\title{
Stability Metrics for Simulation and Flight-Software Assessment and Monitoring of Adaptive Control Assist Compensators
}

\author{
A. S. Hodel* $\quad$ Mark Whorton ${ }^{\dagger} \quad$ J. Jim Zhu ${ }^{\ddagger}$
}

\begin{abstract}
Due to a need for improved reliability and performance in aerospace systems, there is increased interest in the use of adaptive control or other nonlinear, time-varying control designs in aerospace vehicles. While such techniques are built on Lyapunov stability theory, they lack an accompanying set of metrics for the assessment of stability margins such as the classical gain and phase margins used in linear time-invariant systems. Such metrics must both be physically meaningful and permit the user to draw conclusions in a straightforward fashion.

We present in this paper a roadmap to the development of metrics appropriate to nonlinear, time-varying systems. We also present two case studies in which frozen-time gain and phase margins incorrectly predict stability or instability. We then present a multi-resolution analysis approach that permits on-line real-time stability assessment of nonlinear systems.
\end{abstract}

\section{Introduction}

We present in this paper the results of our preliminary investigation into the development and use of stability metrics for stability analysis of adaptive control in aerospace vehicles. The primary outcome of this effort is the development of a road map toward the development and implementation of practical stability metrics. Included in this paper are: an overview of the long-term goals, objectives, and expected outcomes related to the development of practical stability metrics; $\S \mathrm{II}$; and a description of a potential technical approach for the development of stability metrics, §III. This description begins with a literature review of existing metrics, followed by a description of technology gaps and approaches to fill those gaps. We then present preliminary results in our investigation that indicate the failure of current stability metrics (gain, phase margins). These same results also indicate the potential for a multi-resolution analysis (wavelet decomposition). We conclude this discussion with a formal road map toward the development of stability metrics and associated software implementation.

\section{Project overview}

The effort detailed in this paper is the first step to a much larger, long term effort. The goal of this longterm effort is to develop practical theoretic metrics for nonlinear, time-varying systems to facilitate stability and performance analysis and design methods for adaptive/intelligent reconfigurable flight control. These metrics must meet several objectives, which we enumerate in $\S$ A. The outcomes of our road map for the development of these metrics are enumerated in $\S \mathrm{B}$.

\section{A. Objectives}

Theoretical stability metrics within this project should achieve the following objectives.

\footnotetext{
*Department of Electrical and Computer Engineering, 200 Broun Hall, Auburn University, AL 36849-5201. hodelas@auburn.edu (334) 844-1854.

$\dagger$ Marshall Space Flight Center, Huntsville, AL, 35812, mark. whorton@msfc.nasa.gov

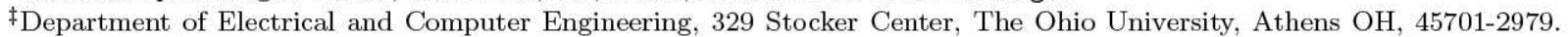
zhuj@ohio.edu (740) 597-1506.
} 
1. Applicable to complex nonlinear, time-varying systems with arbitrary order such as a flight control system;

2. Capable of addressing time-scale separation inherent in different actuators, as well as the nonlinear dynamic nature of the actuator response;

3. Capable of addressing stability and performance robustness as well as transient performance relating the metrics to classical measures such as gain margin, phase margin;

4. Capable of facilitating fault prognosis and diagnosis;

5. Capable of facilitating multi-objective direct, indirect, and hybrid (direct and indirect) adaptive control methods to mitigate multiple faults, failures, and damage conditions under uncertain (and potentially deteriorating) conditions.

6. Appropriate for use in flight software verification and validation $(\mathrm{V} \& \mathrm{~V})$.

7. Capable of balancing speed of response with risk reduction while taking into account the human operator.

\section{B. Expected Outcomes}

Our long-term road map in pursuit of the above objectives is expected to yield the following outcomes:

1. A necessity stability metric called Departure Index (DI) based on Lyapunov's first method;

2. A sufficiency stability metric for equilibrium stability called Stability Margin Index (SMI) based on Lyapunov's second method;

3. A stability fault warning indicator called Stability Fault Warning Indicator (SFWI) based on Lyapunov's second method;

4. A sufficiency stability metric called Passivity Margin (PaM) that is the nonlinear, time-varying counterpart to the linear time-invariant phase margin $(\mathrm{PhM})$ based on passivity theory;

5. A sufficiency stability metric called $\mathcal{L}_{2}$-Gain Margin $\left(\mathcal{L}_{2}\right.$-GM) that is the nonlinear, time-varying counterpart to the linear time-invariant gain margin (GM) based on the small gain theorem and passivity theory.

6. Software implementation and simulation demonstration of the use and effectiveness of the proposed stability metrics in controller adaptation and reconfiguration $(\mathrm{A} \& \mathrm{R})$, fault prediction and detection $(\mathrm{P} \& \mathrm{D})$ and flight software V\&V.

\section{Technical background}

The development of intelligent and adaptive flight control software for resilient aerospace vehicles is a task that is both challenging and essential. This conclusion is based upon several observations:

- Vehicle models are inherently nonlinear, time-varying and complex, therefore model simplification is often used in controller design.

- The vehicle model, environment, and condition. (component conditions may be nominal/degraded/faulty) are uncertain.

- Vehicle tolerances to off-nominal operation (e.g., stall, side slip, spin) may be limited.

- Vehicle control authority is limited and may degrade in off-nominal flight conditions. 
As a result, control flight software must be capable of responding to changing and/or degrading vehicle conditions while taking into account the uncertainties in the vehicle dynamic model and the flight environment. The design of control algorithms that achieve these goals fall into two general categories: robust control and adaptive control.

Robust control refers to a control system's ability to maintain performance and/or stability in the presence of modeling errors, whether parametric, dynamic, or both. In the linear time-invariant setting, singleinput single output systems robustness is commonly assessed in terms of loop gain frequency response characteristics, based on Nyquist's theorem. The simplest and most common quantitative measures in this setting are gain and phase margins. More recently, state-space treatment of robustness includes such theoretical constructs as $\mathcal{H}_{\infty}$ norms and optimal control, ${ }^{1} \mu$-analysis and synthesis, ${ }^{2}$ which provide measures of robustness with respect to specified uncertainty models and connections. Numerous metrics have been defined in an attempt to identify robustness margins, such as the graph metric, ${ }^{3}$ the gap metric,${ }^{4}$ the pointwise gap metric, ${ }^{5}$ and the $\nu$-gap metric, ${ }^{6}$ many of which have been extended to nonlinear systems. These results tend to be conservative, similar to results based on the small gain theorem. ${ }^{7}$

Robust control techniques, particularly those based on linear time-invariant (LTI) system theory, tradeoff system performance for robustness in a deterministic, worst-case optimal manner. They often treat known nonlinear, time-varying (NLTV) dynamics as uncertainties, therefore the resulting control systems, when applied to the NLTV plant, are often either marginal or overly conservative in performance and stability robustness. On the other hand, adaptive control approaches seek optimality in both performance and robustness in response to system behavior change in real time. In principle the adaptive control approaches should achieve improved performance over the LTI robust control methods. However, Cusumano and Poolla ${ }^{8}$ discuss capabilities and limitations of adaptation (NLTV control) vs robust (LTI) control in terms of the nature of system uncertainty: parametric uncertainty yields advantages to NLTV control, but dynamic uncertainty reduces that advantage so that, under some conditions, the achievable performance of robust control will approach that of NLTV control. One cause for this limitation is that most adaptation laws are synthesized based on LTI stability criteria, which do not apply in general to NLTV systems. Many nonlinear adaptive control techniques rely on feedback linearization to cancel the nonlinearity in order to use the LTI stability criteria. However, the cancellation is model based and via feedback, therefore it is sensitive to dynamic uncertainty (singular perturbation). Another challenge faced by adaptive control is the difficulty in software V\&V for nondeterministic behaviors due to on-line adaptation. Some adaptive and fault tolerant control algorithms also require vehicle failure information form parameter changes to remodeling of the vehicle.

As noted above, most of the LTI-theory based gain-scheduling, robust and adaptive control techniques for NLTV systems rely on a frozen-time extension of the LTI stability criteria, either based on the eigenvalues (poles) or the gain and phase margins of the linearized system at a fixed time. Although counter examples have been provided to show that the stability criterion based on the negative definiteness of the frozentime eigenvalues is neither sufficient ${ }^{9}$ nor necessary ${ }^{10}$ for the stability of LTV systems ${ }^{a}$, the success over almost half a century of such control techniques in many nontrivial industrial applications, such as flight control, seems to suggest that these counter-examples may be merely of academic interest. In fact, the widespread success of frozen-time based control techniques can largely be attributed to a (rigorously proven) slowly varying constraint, under which the frozen-time eigenvalue stability provides a sufficiency criterion for stability. As the demands grow for agility and fast transition through wide operating conditions, the frozentime based control techniques face increasing challenges. Indeed, the modern robust control techniques were developed in the hope of expanding LTI based techniques in the presence of NLTV dynamics that are, for the convenience of the LTI control techniques, purposely ignored in frozen-time, frozen-state modeling.

Here we present two practical examples of NLTV systems to show that blind trust and application of frozen-time analysis may result in an unreliable stability assessment. Both examples can be modeled as pendulums with time-depending damping and stiffness coefficients. Thus, they have broad implications since many practical dynamical systems can be decomposed into sets of second-order modes. These examples are used later in this paper to demonstrate the effectiveness of our proposed stability metrics based on Lyapunov's first method.

Example 1. Playground Swing A playground swing can be modeled as a pendulum with a time-varying length as the player pumps the swing up by moving the torso up and down, as shown in Figure 1. The

\footnotetext{
${ }^{a}$ See also, ${ }^{11}$ where the original Marcus-Yamabe and Wu equations are parameterized to demonstrate the parameter set wherein the frozen-time stability criterion fails to be sufficient or necessary for stability assessment.
} 


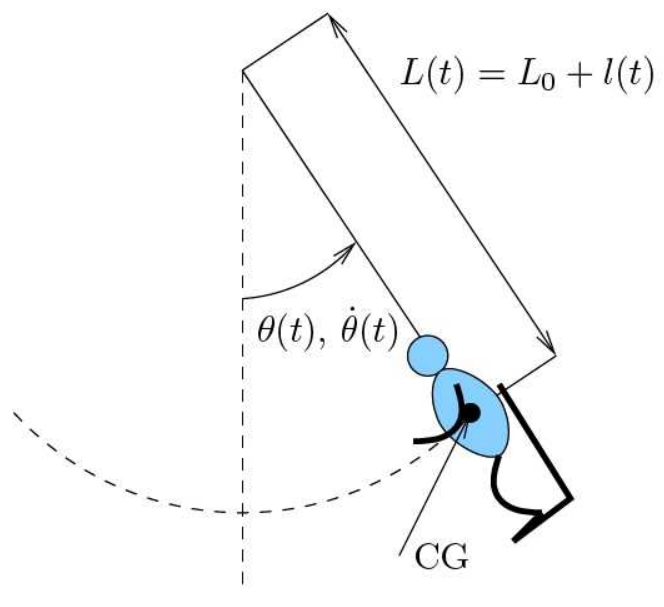

Figure 1. Playground swing: $m=20(\mathrm{~kg}), g=9.81\left(\mathbf{m} / \mathrm{s}^{2}\right), c=20\left(\mathrm{~kg} \cdot \mathrm{m}^{2} / \mathbf{s}\right), L_{0}=3.0(\mathbf{m}),-0.2 \leq l(t) \leq 0.2(\mathbf{m})$.

Table 1. Frozen-time analysis of the playground swing (10 cases)

\begin{tabular}{|c|c|c|c|c|}
\hline Frozen Length & \multicolumn{2}{|c|}{ Frozen-time Poles } & \multicolumn{2}{c|}{ Frozen-time Margins } \\
\hline$L+l(t)$ & $\zeta$ & $\omega$ & Gain Margin & Phase Margin \\
\hline 2.800 & 1.942 & 0.038 & $\infty$ & 13.755 \\
\hline 2.844 & 1.910 & 0.036 & $\infty$ & 13.217 \\
\hline 2.889 & 1.879 & 0.035 & $\infty$ & 12.709 \\
\hline 2.933 & 1.850 & 0.033 & $\infty$ & 12.228 \\
\hline 2.978 & 1.822 & 0.031 & $\infty$ & 11.773 \\
\hline 3.022 & 1.795 & 0.030 & $\infty$ & 11.342 \\
\hline 3.067 & 1.769 & 0.029 & $\infty$ & 10.932 \\
\hline 3.111 & 1.745 & 0.028 & $\infty$ & 10.543 \\
\hline 3.156 & 1.721 & 0.027 & $\infty$ & 10.173 \\
\hline 3.200 & 1.700 & 0.026 & $\infty$ & 9.821 \\
\hline
\end{tabular}

"frozen-time" equation of motion is given by fixing the length of the pendulum at every moment in time as

$$
\ddot{\theta}+\frac{c}{m[L+l(t)]^{2}} \dot{\theta}+\frac{g}{[L+l(t)]} \sin (\theta)=0
$$

Clearly, for every fixed length $l(t)$, the equilibrium point $(\theta(t), \dot{\theta}(t)) \equiv(0,0)$ is asymptotically stable. Figure 2 shows the impulse responses and frequency responses of the frozen-time model (1) for ten different fixed lengths. Table 1 shows the frozen-time eigenvalues and the frozen-time gain and phase margins. All plots and data indicate asymptotic stability. However, the correct equation of motion is given by the principle of conservation of angular momentum as

$$
\ddot{\theta}+\left(\frac{2 \dot{l}(t)}{L+l(t)}+\frac{c}{m[L+l(t)]^{2}}\right) \dot{\theta}+\frac{g}{[L+l(t)]} \sin (\theta)=0
$$

Using the parameters shown in Figure 1 and a pumping motion $l(t)=0.2 \operatorname{sat}(-\dot{\theta}(t) \theta(t)$ ), the null equilibrium $(0,0)$ can be destabilized with a sizable damping coefficient $c>0$, as shown in Figure 3. 


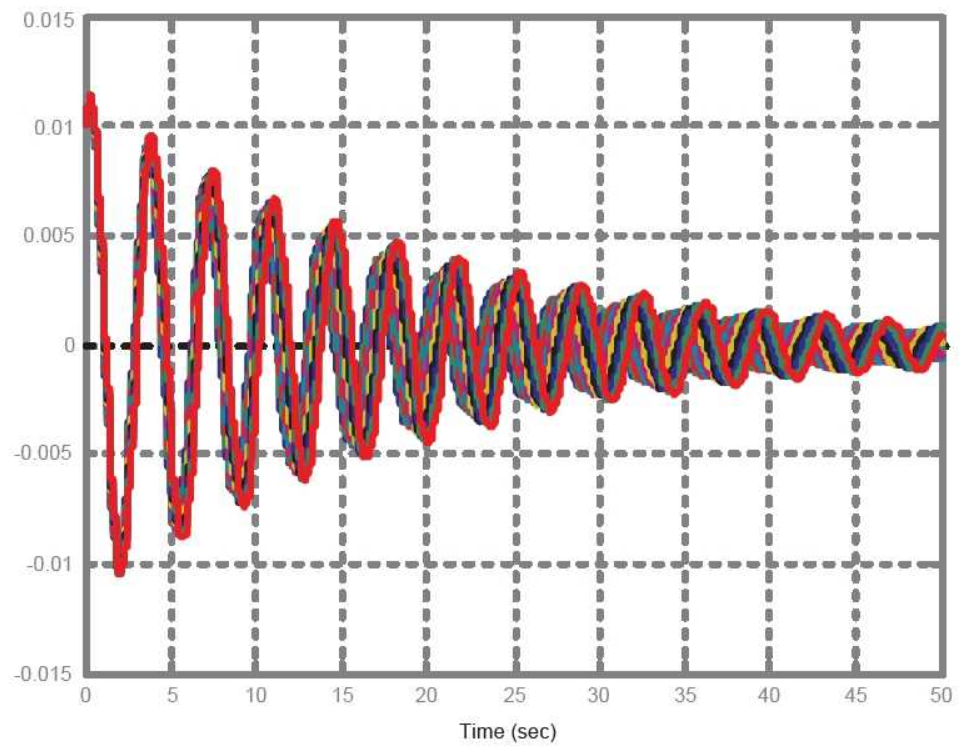

(a) Impulse responses

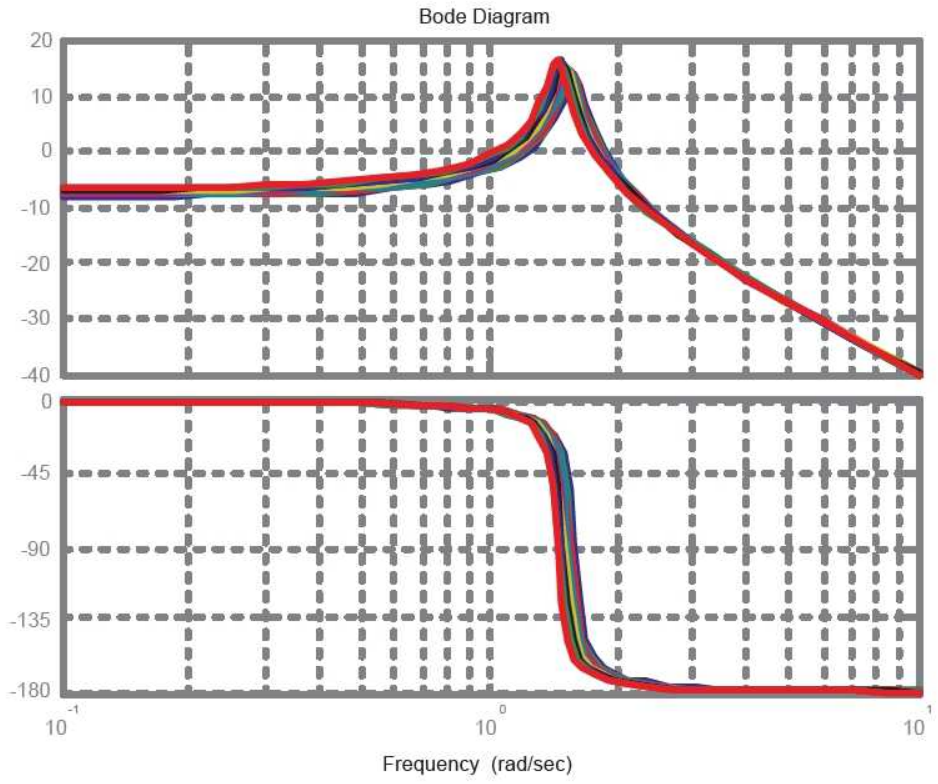

(b) Frequency responses

Figure 2. Frozen-time analysis of the playground swing (10 cases)

This example convincingly shows that the frozen-time stability criterion fails to be a sufficiency condition for stability of LTV and linearizable NLTV systems, and the frozen-time gain margin and phase margin are unreliable stability metrics for LTV and linearizable NLTV systems. Noticing that the frozen-time model 


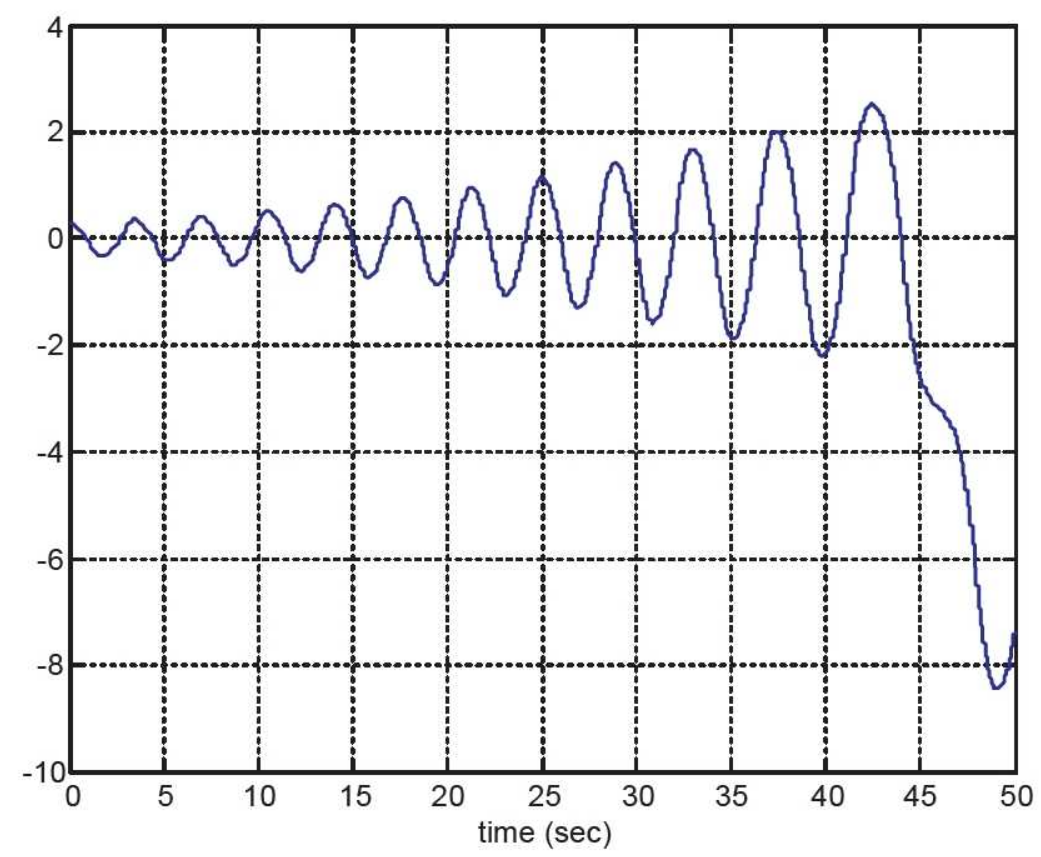

(a) Playground swing angle $\theta(t)$ destabilized by pumping motion

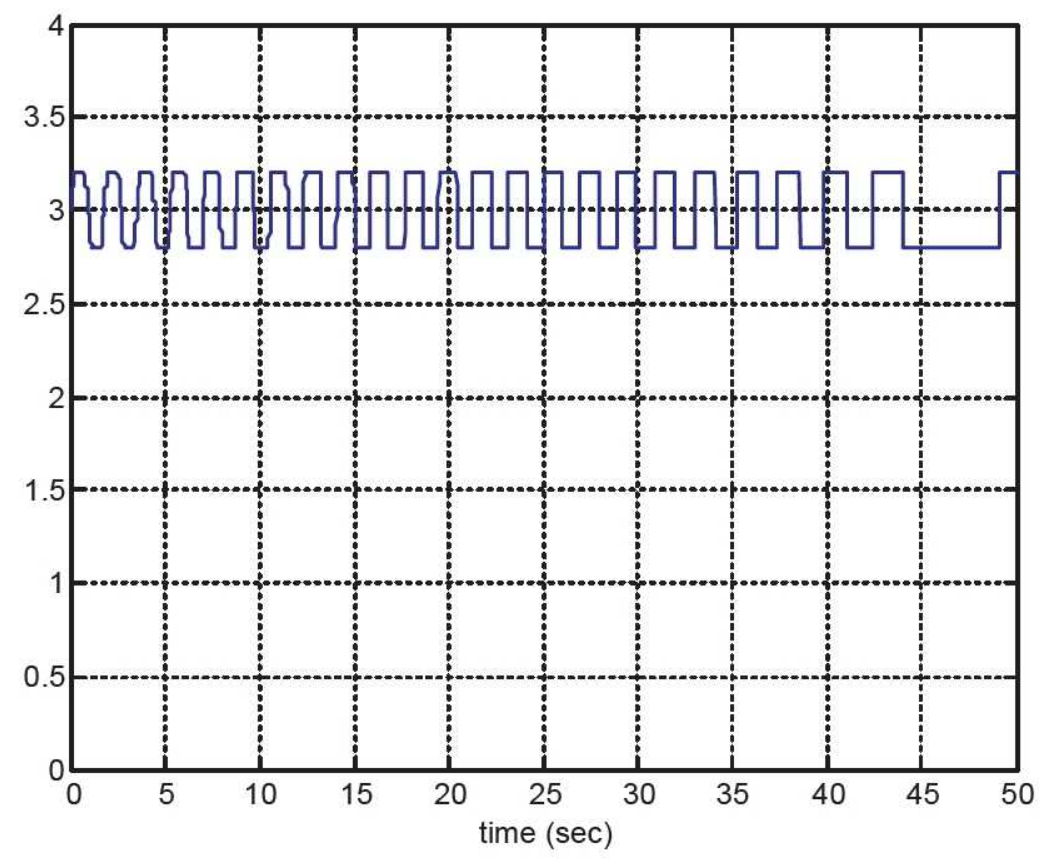

(b) Swing length $L+l(t)$ as a result of the pumping motion

Figure 3. Nonlinear simulation of the playground swing model (2).

(1) ignored the $\dot{l}(t)$ term, which becomes negative at times, one may argue that if the frozen-time analysis is applied to the correct model (2), the instability may have been correctly predicted. The next practical example convincingly shows that, to the contrary, a LTV system may be asymptotically stable even if its frozen-time model is unstable at times, thereby showing that the frozen-time stability criterion fail to be a 


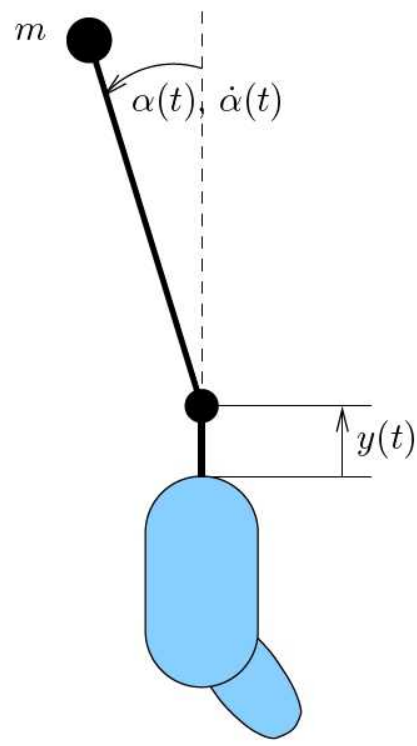

Figure 4. Jigsaw mounted inverted pendulum: $m=0.5(\mathrm{~kg}), g=9.81\left(\mathrm{~m} / \mathrm{s}^{2}\right), c=0.01\left(\mathrm{~kg} \cdot \mathrm{m}^{2} / \mathrm{s}\right), L=0.5(\mathbf{m})$, $y(t)=A \cos (\omega t)$, where $A=0.01(\mathbf{m}), \omega=314(\mathrm{rad} / \mathrm{s})$

necessity condition for LTV and NLTV systems.

Example 2. (Inverted Pendulum on a Vertically Vibrating Base) An inverted pendulum mounted on a vertically vibrating base, such as a jigsaw, is shown in Figure 4. The "frozen-time" equation of motion for the inverted pendulum on a vertically vibrating base is no difference from an inverted pendulum mounted on a fixed base

$$
\ddot{\alpha}+\frac{c}{m L^{2}} \dot{\alpha}+\left(-\frac{g}{L}\right) \sin (\alpha)=0
$$

Therefore the upright equilibrium point $(\alpha(t), \dot{\alpha}(t)) \equiv(0,0)$ is unstable. However, the correct equation of motion is given by the conservation of angular momentum as

$$
\ddot{\alpha}+\frac{c}{m L^{2}} \dot{\alpha}+\left(-\frac{g}{L}+\frac{\ddot{y}(t)}{L}\right) \sin (\alpha)=0
$$

where $y(t)$ is the vertical displacement of the base. Now we consider the frozen-time analysis with the effect of the moving base with ten different acceleration values $\ddot{y}(t)$ for $y(t)=0.01 \cos (314 t)$, which amounts to 3,000 strokes per minute with a stroke displacement of 0.02 meters. The frozen-time eigenvalues and gain, phase margins are shown in Table 2. The corresponding impulse responses and frequency responses are shown in Figure 5. These results show that in five out of the ten cases the frozen-time criterion predicts instability. Thus it seems reasonable to predict the system would be unstable.

By letting $y(\tau)=A \cos (2 \tau)$ and $\mathrm{d} / \mathrm{d} \tau=\frac{1}{\omega} \mathrm{d} / \mathrm{d} t$, where $\omega$ is the angular frequency of the base vibration, and $\tau=\omega t$ is the normalized time, equation (4) linearizes to a damped Mathieu equation

$$
\ddot{x}+2 \zeta \dot{x}+(a-2 q \cos (2 \tau)) x=0,
$$

whose stability domain is well known ${ }^{12}$ and is given by Figure 6 . It is seen that under a certain parameter combinations of $a, q$, the upright equilibrium can be stabilized. Figures 7 shows the simulation result for the parameters given in Figure 4, which indeed stabilizes the null equilibrium. This example convincingly demonstrates the failure of frozen-time criteria as a necessity condition for stability.

Stability and robustness in nonlinear systems are typically addressed in terms of Lyapunov analysis, passivity and the small gain theorem. ${ }^{13-15}$ Very roughly speaking, the small gain theorem corresponds 
Table 2. Frozen-time analysis of the jigsaw mounted inverted pendulum (10 cases)

\begin{tabular}{|c|c|c|c|c|}
\hline Frozen Base Accel. & \multicolumn{2}{|c|}{ Frozen-time Poles } & \multicolumn{2}{c|}{ Frozen-time Margins } \\
\hline$\ddot{y}\left(\mathrm{~m} / \mathrm{s}^{2}\right)$ & $\lambda_{1}$ & $\lambda_{2}$ & Gain Margin & Phase Margin \\
\hline-985.96 & -44.6667 & 44.5867 & N/A & N/A \\
\hline-766.86 & -39.4524 & 39.3724 & N/A & N/A \\
\hline-547.76 & -33.4336 & 33.3536 & N/A & N/A \\
\hline-328.65 & -26.0578 & 25.9778 & N/A & N/A \\
\hline-109.55 & -15.4907 & 15.4107 & N/A & N/A \\
\hline 109.55 & $-0.04+14.1238 i$ & $-0.04-14.1238 i$ & 0.9801 & 0.0065 \\
\hline 328.65 & $-0.04+25.2524 i$ & $-0.04-25.2524 i$ & 0.9362 & 0.0116 \\
\hline 547.76 & $-0.04+32.8008 i$ & $-0.04-32.8008 i$ & 0.8924 & 0.015 \\
\hline 766.86 & $-0.04+38.9114 i$ & $-0.04-38.9114 i$ & 0.8486 & 0.0178 \\
\hline 985.96 & $-0.04+44.1848 i$ & $-0.04-44.1848 i$ & 0.8048 & 0.0203 \\
\hline
\end{tabular}

to the concept of a gain margin and passivity corresponds to the concept of phase margin. However, it appears that these theoretical stability criteria have not been reduced to practical measures that can be used in control $A \& R$, fault $\mathrm{P} \& \mathrm{D}$ and control software $\mathrm{V} \& \mathrm{~V}$. Most recently, Zhu et al ${ }^{16}$ demonstrated the use of Lyapunov's first method in the development of a simple metric for analysis and on-line detection of instability, where they used an empirical technique to determine nonlinear measures analogous to gain and phase (delay) margins. The authors prior work in ${ }^{16}$ also alludes to the development of additional practical stability metrics using Lyapunov second method, passivity and L-stability theory under a NASA contract for the NRA8-30 Space Launch Vehicle Program that was subsequently aborted.

\section{Approach}

The classical stability indices such as the PhM and GM for LTI systems rely on Laplace or Fourier transforms that are not applicable to LTV and NLTV system. Therefore, in general these concepts cannot be extended to LTV and NLTV systems using frequency domain or frozen-time approach. Our long term goal is to develop practical theoretic stability metrics for NLTV system (including LTV systems as a subset) based on Lyapunov's first and second methods for stability assessment,${ }^{17}$ and the modern nonlinear stability theories such as the passivity theory and the $\mathcal{L}$-stability theory (see, e.g. Khalil ${ }^{13}$ ) .

\section{Lyapunov Methods for Assessing Stability of Motion}

Consider the general NLTV system described by state equations

$$
\dot{x}=f(t, x), \quad x\left(t_{0}\right)=x_{0}, \quad t \geq 0
$$

where $f$ is assumed to be piecewise continuous in $t$ and locally Lipschitz in $x$. A state vector $x_{e}(t) \in \mathbb{R}^{n}$ is called an equilibrium state of (6) if $f\left(t, x_{e}\right) \equiv 0$. An equilibrium point $x_{e}$ is said to be isolated if there exists a $\delta>0$ such that for any other equilibrium point $\tilde{x}_{e}$ of $(6),\left\|x-\tilde{x}_{e}\right\|>\delta$. In control systems, (6) may model the set-point or tracking error dynamics of a closed-loop system, for which the origin is always an equilibrium (the null equilibrium) that we wish to attain and maintain under perturbations to the trajectories of the system. Thus, it is of interest to determine the stability of the null equilibrium in the sense of Lyapunov. It is noted that when the system is time-varying, which is the case for adaptive control systems, uniformity of the stability and asymptotic stability must be considered. In order to achieve inherent robustness to regular and singular perturbations of the system (structural stability, or robust stability), and boundedinput-bounded-output response to disturbances and noises (or $\mathcal{L}_{\infty}$-stability), (uniform) exponential stability should be designed for and maintained, even if only locally (see, e.g. Khalili ${ }^{13}$ Chapters $4,5,9,11$ ). 


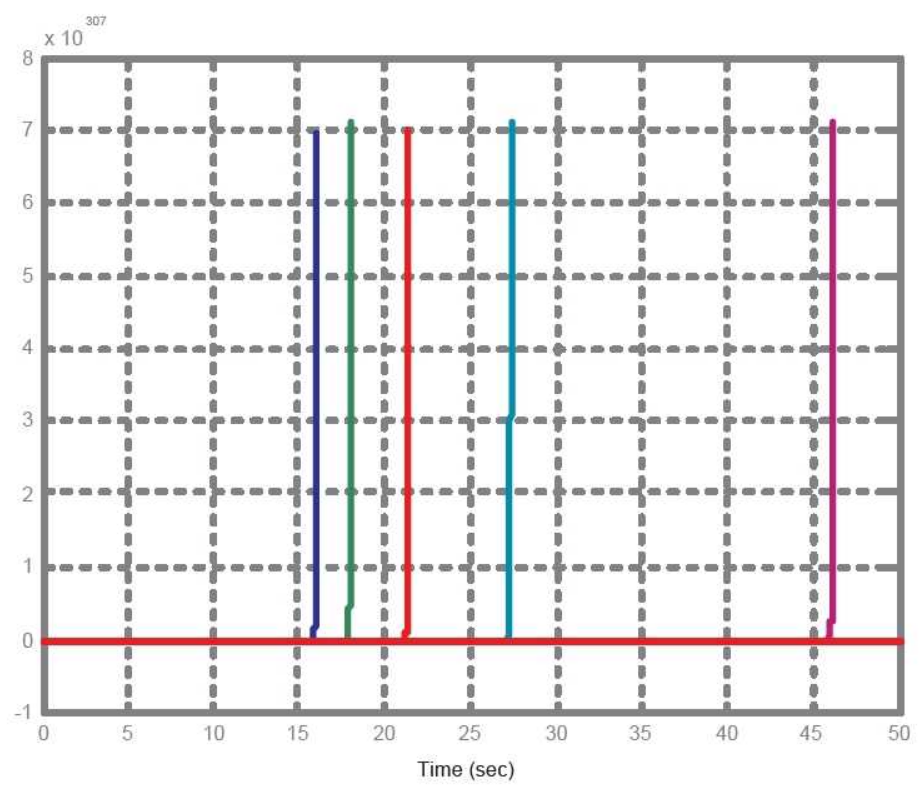

(a) Impulse responses

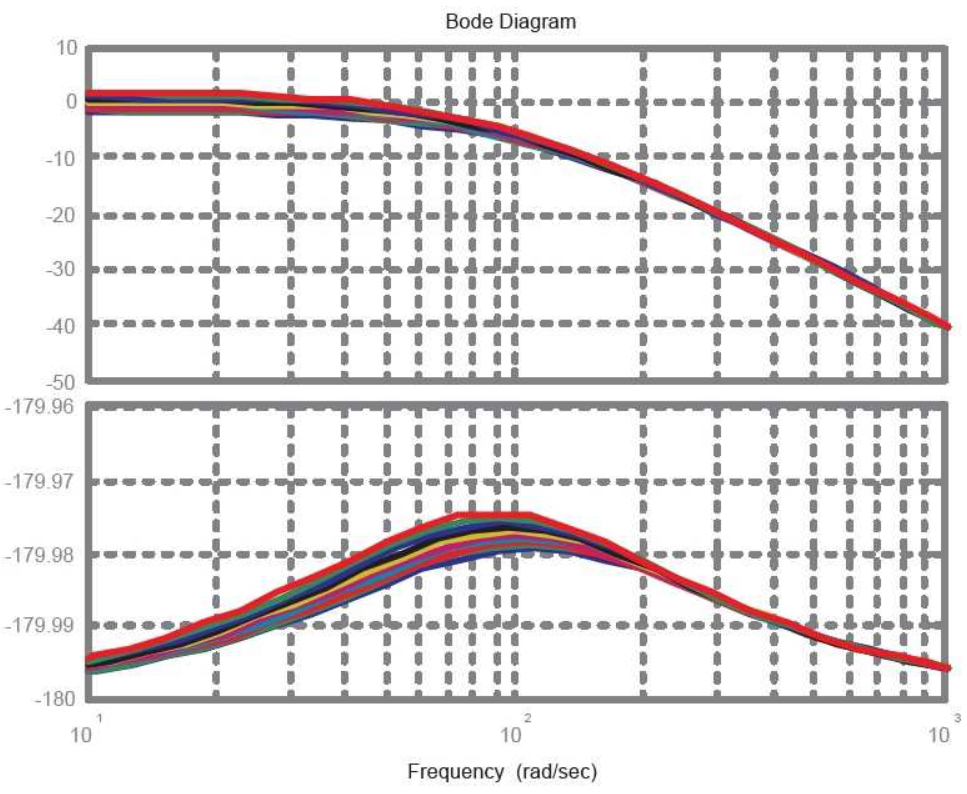

(b) Frequency responses

Figure 5. Frozen-time analysis of the playground swing (10 cases)

In addition to defining in a rigorous manner the concept of stability of motion, Lyapunov also devised two methods for the assessment of stability of nonlinear motion. These two methods are illustrated below with the most elementary cases for the null equilibrium. 

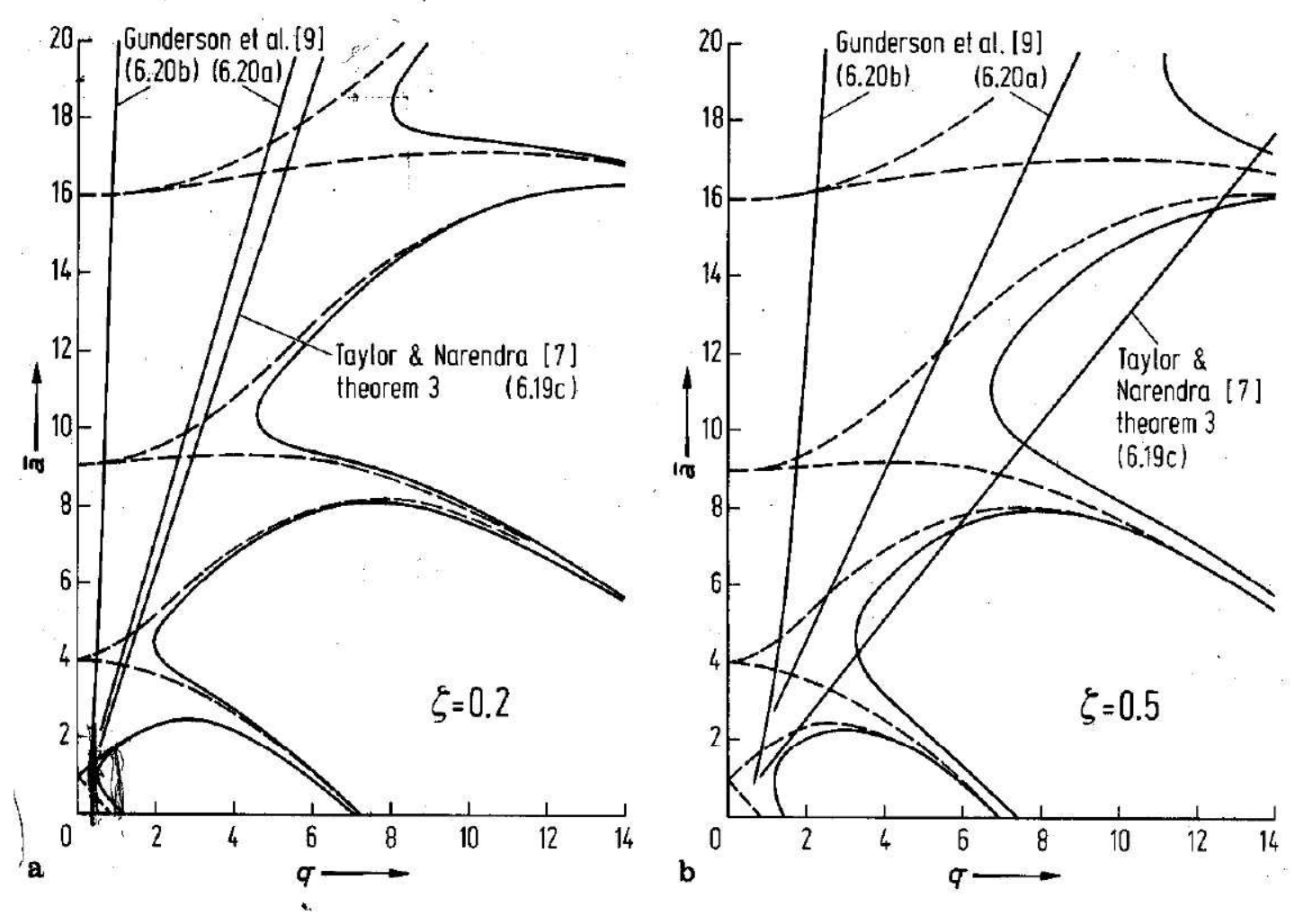

Figure 6. Stability Domain of the Damped Mathieu Equation ${ }^{12}$.

1. Lyapunov's first method (indirect method): define the Lyapunov exponent for a solution $x(t)=s\left(t, x_{0}, t_{0}\right)$

$$
\lambda\left(x_{0}, t_{0}\right)=\limsup _{t \rightarrow \infty} \frac{\ln \left\|s\left(t, x_{0}, t_{0}\right)\right\|}{t}
$$

Then $x(t) \rightarrow 0$ exponentially as $t \rightarrow \infty$ if $\lambda\left(x_{0}, t_{0}\right)<0$. Thus, the origin is asymptotically stable at $t_{0}$ if $\lambda\left(x_{0}, t_{0}\right)<0$ for all $\left\|x_{0}\right\|<\gamma$, and it is (uniformly) exponentially stable on $\left[T_{0}, \infty\right)$ if $\lambda\left(x_{0}, t_{0}\right)<$ $-c<0$, for some $c>0$, for all $\left\|x_{0}\right\|<\gamma$ and for all $t_{0} \in\left[T_{0}, \infty\right)$.

2. Lyapunov's second method (direct method): for an autonomous (time-invariant) nonlinear system (6), i.e. $\dot{x}=f(x)$, the origin is asymptotically stable if one can find a positive definite, continuously differentiable function $V(x(t))$ such that $\dot{V}(x(t))$ is negative definite (in a neighborhood of the null equilibrium). The function $V(x)$ is called a Lyapunov function for (6) at the null equilibrium.

It is noted that the second method for NLTV systems may require a time-dependent Lyapunov function $V(t, x)$, in which case three time-independent positive definite functions $W_{1}(x), W_{2}(x)$ and $W_{3}(x)$ are required such that

$$
\begin{aligned}
W_{1}(x) & \leq V(t, x) \leq W_{2}(x) \\
\dot{V}(t, x(t)) & =\frac{\partial V}{\partial t}+\frac{\partial V}{\partial x} f(t, x) \leq-W_{3}(x)
\end{aligned}
$$

Moreover, for exponential stability, it is required that $W_{i}(x)=k_{i}\|x\|^{a}$ for some positive constants $a$ and $k_{i}$, $i=1,2,3$. The second method is a sufficiency condition in that the inability to find a Lyapunov function does not imply the equilibrium is unstable.

It is also noted that Lyapunov's first method is often mistaken as the linearization method in the literature and many textbooks. In fact, the first method was only applied by Lyapunov to LTI and LTV systems to 


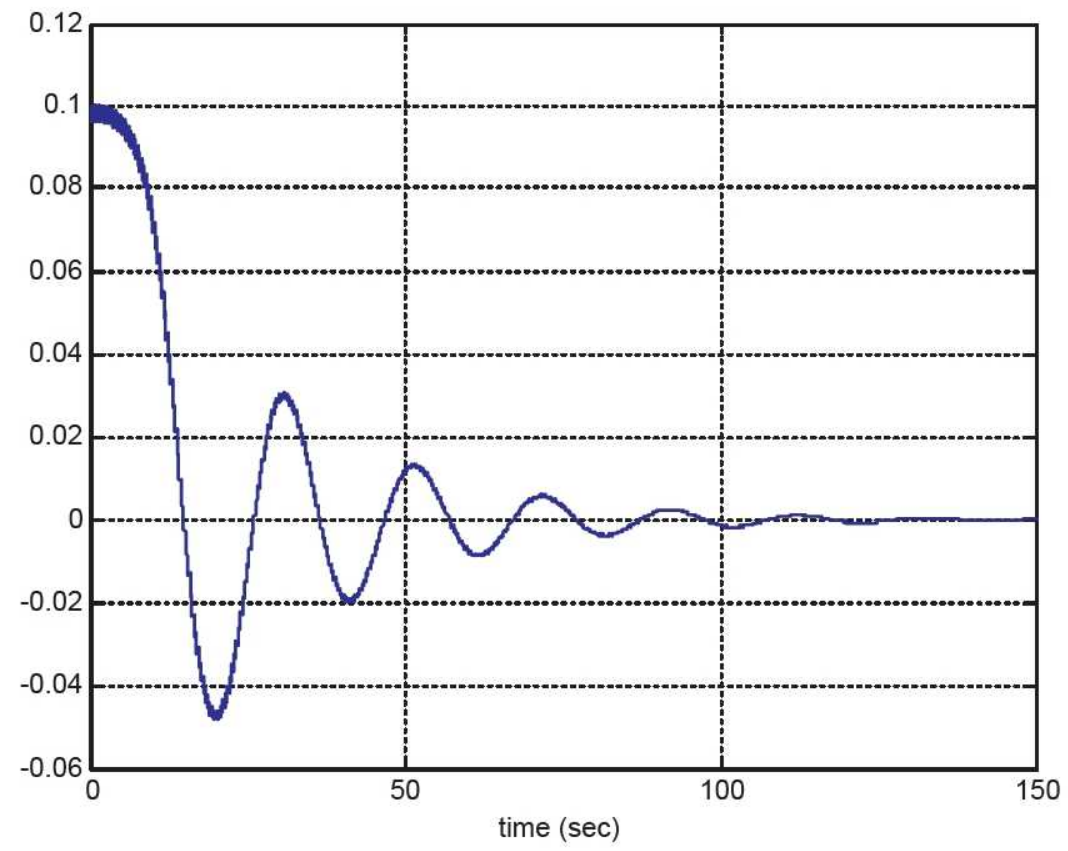

Figure 7. Inverted pendulum stabilized by the pumping motion at the base

derive some general results. The linearization method, which is based on the important equivalence condition that the null equilibrium of a NLTV system (6) is exponentially stable if and only if the linearized system is exponentially stable, is established using the second method.

\section{Lyapunov First Method Based Stability Metrics}

While the Lyapunov exponent for a particular (tracking error) trajectory $x(t)=s\left(t, x_{0}, t_{0}\right)$ is readily computable in real-time by

$$
\lambda_{0}=\limsup _{t \rightarrow \infty} \frac{\int_{t_{0}}^{t} \frac{\|\dot{x}(\tau)\|}{\|x(\tau)\|} d \tau}{t-t_{0}}
$$

for some convenient norm and its negativity can be monitored, a difficulty in using the first method in assessing stability is that it requires the solution of the nonlinear system for every possible initial condition. However, this does not prevent it from being used as an instability assessment for NLTV systems. Another difficulty is that the exponent is defined for $t \rightarrow \infty$, while any practical flight mission is of finite duration. For time-dependent systems, the system trajectory may diverge, also known as departure, momentarily so as to violate some operational constraints, even though it may ultimately converge to zero as $t \rightarrow \infty$. In order to detect such practical instability, a short-time, or windowed, Lyapunov exponent can be defined as

$$
\lambda_{T}(t)=\frac{\int_{t-T}^{t} \frac{\|\dot{x}(\tau)\|}{x(\tau)}}{T} d \tau
$$

where $T$ is the width of the moving window over which the exponential departure rate is observed. In the sequel, we shall call $\lambda_{0}$ a Long-term Departure Index (LDI) for the trajectory, and $\lambda_{T}$ a Short-term Departure Index (SDI). Being necessity conditions for stability, the main utility of this method is to facilitate on-line warning of onset of loss of stability. Figure 8 shows an implementation of the LDI/SDI described above in a tracking control system, where $x(t)$ is the tracking error. It is noted that the magnitude of the DI $\left|\lambda_{T}\right|$ can 


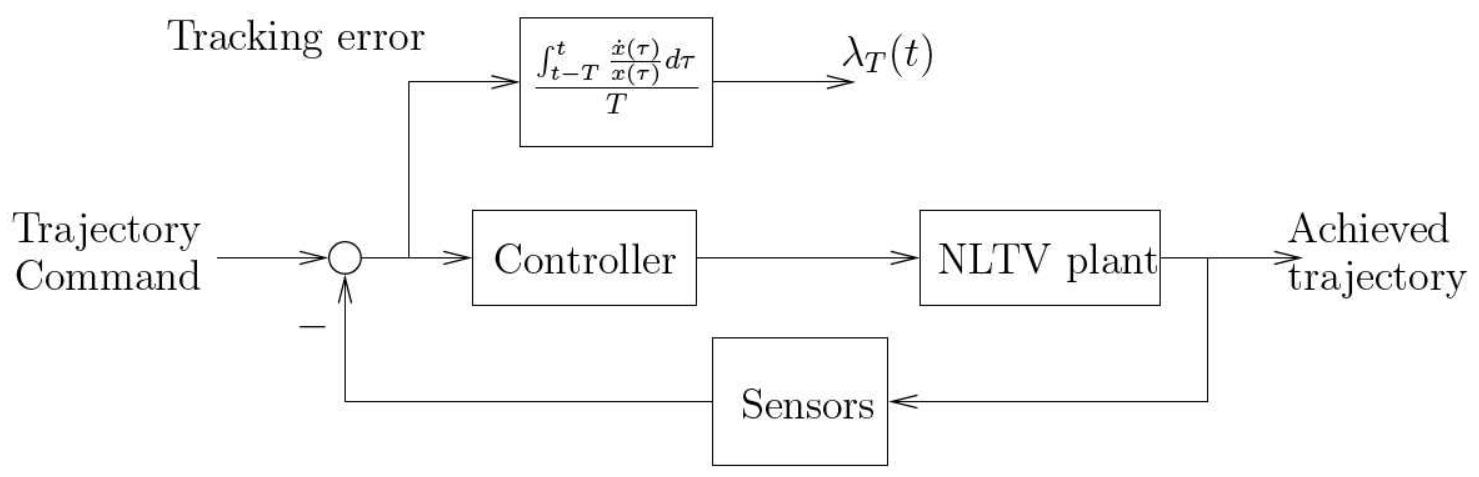

Figure 8. Implementation of the DI.

be used as a transient performance indicator as it is the exponential decay rate of the (tracking error) state variable.

The LDI and the SDI have been implemented and the effectiveness of the DIs has been studied under NASA NRA8-30 for reusable launch vehicles on a high fidelity 6-DOF simulation platform MAVERIC at Marshall Space Flight Center. Very encouraging preliminary results were reported Best et al. ${ }^{16}$ In this paper, we apply this method to aircraft adaptive flight control systems to verify it's effectiveness and to address some of the issues identified in the previous study. In particular, the method has a technical difficulty in coping with trajectory zero-crossings of a single state variable. This problem can be solved in theory using Hilbert transform. However, the Hilbert transform is an anti-causal process and cannot be realized in real-time for nonstationary (time-varying) processes. Our preliminary suggestion for this method for circumventing this difficulty is to use short-time, narrow-band (wavelet) approximations to the Hilbert transform, which can be implemented in real-time using a bandpass filter bank. The theoretical study will focus on approximation error analysis and validation of the concept and methodology. Use of the modal information provided by the wavelet analysis for diagnostic and prognostic of flight condition and vehicle health, and for control adaptation will also be investigated.

\section{Lyapunov Second Method Based Stability Metrics}

In addition to assessing stability of an equilibrium state, the second method of Lyapunov also offers a (conservative) estimate of the Domain of Attraction with the ensuing Lyapunov function $V(t, x)$, wherein trajectories are guaranteed to converge to the equilibrium. The DoA is specified by the interior of a closed level contour $\Gamma_{c}=\left\{x: W_{1}(x) \equiv C>0\right\}$ wherein $-W_{3}(x)<0$. A practical sufficiency stability metric can be defined as the distance of the current (tracking error) state trajectory $x(t)$ from the boundary of the DoA

$$
\operatorname{dist}\left(x(t), \Gamma_{c}\right)=\inf _{z \in \Gamma_{\mathrm{c}}}\|x(t)-z\|
$$

using a suitable norm, which will be termed Stability Margin Index (SMI), which can be monitored with full state measurement or estimate.

This method depends on an a priori estimate of Domain of Attraction (DoA) using a Lyapunov function for the closed-loop system, which is usually available for controllers designed using Lyapunov's second method. Otherwise an estimate of the DoA may be obtained using an iterative method to construct a Lyapunov function, ${ }^{18}$ or by reverse-time simulation method. The limitation of this method is that it is a model based method. Thus its accuracy and validity rely on the fidelity of the model, and models for adverse flight conditions and vehicle failures need to be developed in order to develop the corresponding estimate of DoA.

As a byproduct of this method, another index to monitor is the negativity of the time derivative of the Lyapunov function along the current trajectory. Although the negativity alone is neither a necessary nor a sufficient condition for stability, loss of negativity in an a priori validated DoA can be used as an indicator for abnormal flight conditions or vehicle health. Moreover, the magnitude $|\dot{V}(x)|$ is an indicator of transient performance when $\dot{V}(x)<0$, and the magnitude $|V(x)|$ is an indicator of steady-state performance indicator. 


\section{Assessment of Input-Output Stability and Stability of Feedback Loops}

Consider a NLTV control system described by

$$
\begin{gathered}
\dot{x}(t)=f(x(t), u(t), \theta(t)) \\
y(t)=h(x(t), u(t), \theta(t))
\end{gathered}
$$

where $x(t) \in \mathbb{R}^{n}, u(t) \in \mathbb{R}^{l}, y(t) \in \mathbb{R}^{m}$ are the state, input and output vectors, respectively, and $\theta(t) \in \mathbb{R}^{p}$ is a piecewise continuous time-varying parameter vector representing time-dependent flight condition parameters such as Mach and dynamic pressure, system parameters such as aerodynamic coefficients, and adaptive controller parameters (gains). The function $f$ is locally Lipschitz and $h$ is continuous. The NLTV system (7) defines an input-output (I/O) map $y=H u$, which can be used to model the subsystems in a feedback loop, such as the plant, actuators, sensors and controllers. It is noted that the overall closed-loop system with a reference input is itself an I/O map of the form (7).

It is of practical interest to consider whether the output response $y=H u$ to every norm-bounded input $u$ is norm-bounded for some suitable norms. If so, the I/O map $H$ is said to be input-output stable with respect to the norms of the I/O signals. Commonly used norms in this context are the extended $\mathcal{L}_{p}$ norms (for definition, see e.g. Khalil ${ }^{13}$ pp.195-197). In particular, if for every $\mathcal{L}_{p}$ norm-bounded input signal $u$, the system (7) satisfies

$$
\frac{\|H u\|_{\mathcal{L}_{p}}}{\|u\|_{\mathcal{L}_{p}}} \leq \gamma
$$

for some positive constant $\gamma$ then I/O map $H$ is said to be (zero-bias) finite-gain $\mathcal{L}_{p}$-stable, and the supremum of $\gamma$ taken over the entire extended $\mathcal{L}_{p}$-space of the input signals is called the gain of the I/O map. We will subsequently use $\gamma$ to denote the gain of the I/O map $H$. Two commonly used $\mathcal{L}_{p}$ norms are $\mathcal{L}_{2}$ and $\mathcal{L}_{\infty}$, representing the energy and peak-magnitude of the signal over a time-interval. Thus finite-gain $\mathcal{L}_{2}$-stability signifies bounded-energy I/O stability, and the finite-gain $\mathcal{L}_{\infty}$-stability is the generalization of the BoundedInput-Bound-Output (BIBO) stability for LTI systems, the latter is equivalent to (exponential) asymptotic stability of a LTI system.

It is noted that, unlike LTI systems, for nonlinear systems, the null equilibrium in the (tracking error) state space being merely asymptotically stable does not guarantee I/O stability. The practical implication is that a bounded disturbance or noise input may cause the system output departure. An important result in the $\mathcal{L}_{p}$-stability theory is that, under some mild conditions on $f$ and $h$, exponential stability implies (small-signal) finite-gain $\mathcal{L}_{p}$-stability of the I/O map.

An important $\mathcal{L}_{p}$-stability result is the small-gain theorem for a feedback loop shown in Figure 9, which states that the I/O map from any input to any output of the feedback configuration is finite-gain $\mathcal{L}_{p^{-}}$stable if the loop gain $\gamma=\gamma_{1} \gamma_{2}<1$, where $\gamma_{1}, \gamma_{2}$ are the $\mathcal{L}_{p}$-gains for the subsystems $S_{1}, S_{2}$, respectively. This result can be viewed as a nonlinear extension of the LTI gain margin concept where for stability the frequency response loop gain is required to be less than 1 above the crossover frequency. Thus the margin $\gamma-1$ can be viewed as an $\mathcal{L}_{p^{\text {-gain }}}$ margin.

There are two practical difficulties in applying the $\mathcal{L}_{p}$-gain margin concept in real-time stability assessment. First, it is impractical to estimate the $\mathcal{L}_{p}$-gain in real-time for NLTV systems. Second, the small-gain theorem is an overly restrictive sufficiency condition that precludes high-gain control for improved steadystate precision. In fact, for LTI systems, the loop gain can be greater than unity below the crossover frequency, provided that the phase margin is positive. Both of these difficulties can be circumvented by the passivity theory for NLTV systems.

In a nutshell, the passivity theory is an energy based approach. A system is passive if the power dissipation by the system is greater than or equal to the rate of exchange of stored energy in the system, which can be stated as

$$
u(t)^{\mathrm{T}} y(t) \geq \dot{V}(t, x(t))=\frac{\partial V}{\partial t}+\frac{\partial V}{\partial x} f(t, x)
$$

where $V(t, x)$ is a continuously differentiable positive definite function known as a storage function. The system is said to be strictly passive if the inequality holds strictly, in which case the margin

$$
\psi(x)=u(t)^{\mathrm{T}} y(t)-\dot{V}(t, x(t))>0, \quad \forall x \neq 0
$$




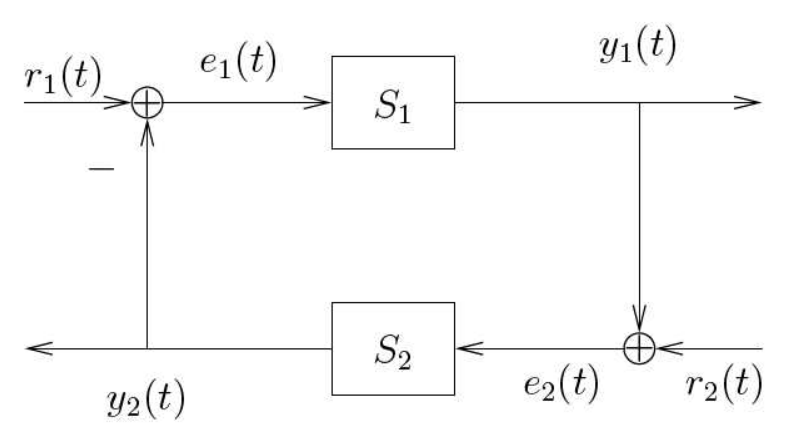

Figure 9. Feedback configuration of two I/O maps.

is known as the excess of passivity. The excess of passivity for the open-loop I/O map $e_{1} \rightarrow y_{2}$ in the feedback configuration shown in Figure 9 can be viewed as a NLTV extension of the concept of phase margin for LTI systems, because the cascade of two strictly passive LTI systems has a positive phase margin. The passivity concept can be extended by considering I/O maps that can be rendered passive via a local feedback

$$
u(t)^{\mathrm{T}} y(t) \geq \dot{V}(t, x(t))+y^{\mathrm{T}} \rho(y), \quad y^{\mathrm{T}} \rho(y)>0, \forall y \neq 0
$$

which is called output strictly passive. The main result of the passivity theory is that the closed-loop I/O map is $\mathcal{L}_{2}$-stable, and the null equilibrium of the zero-input closed-loop system is asymptotically stable if both subsystems are strictly passive, or one or both are output strictly passive with an additional requirement of being zero-state observable, a mild condition that can be satisfied with practical sensors. In addition, an estimate of the $\mathcal{L}_{2}$-gain can be obtained from real-time measurement of the stored energy in the state variables, and the output signals (see, e.g., [13, Ch. 6].

\section{Passivity Theory Based Stability Metrics}

We propose to use the excess of passivity of the open-loop I/O map, called Passivity Margin (PaM), as a NLTV extension to the LTI phase margin concept, and use the margin of the $\mathcal{L}_{2}$-loop-gain from unity, called $\mathcal{L}_{2}$-Gain Margin $\left(\mathcal{L}_{2}\right.$-GM) as a NLTV extension to the LTI gain margin concept. Both margins can be monitored using real-time measurement or estimate of the state variables, and input and output signals. Parasitic energy stored in the neglect fast modes, e.g. actuator dynamics, structural or aeroelasticmodes, will be accommodated by the passivity margins being monitored. Additional modes can be added to the storage function as needed through experimentation to improve fidelity. A sudden loss of passivity margin would imply onset of adverse flight condition, excitation of unmodeled modes or vehicle faults that either inject additional energy into the system, or prevent them from being damped out. Thus, the real-time measurement of these margins can be used for fault $\mathrm{P} \& \mathrm{D}$ and control $\mathrm{A} \& \mathrm{R}$. In particular, the energy measurement can be done in divided frequency bands to aid fault analysis and adaptive control law synthesis using, for example, the time-varying bandwidth filters (TVBF) or time-varying notch filters (TVNF) developed by Zhu et. al. ${ }^{19,20}$ Similar to the Lyapunov second method, the magnitude $|\dot{V}(x)|$ is an indicator of transient performance when $\dot{V}(x)<0$, and the magnitude $|V(x)|$ is an indicator of steady-state performance indicator. Moreover, the integral of $u(t)^{\mathrm{T}} y(t)$ from the control input to the plant output over a time interval is an indicator of the control energy consumption over that time interval.

\section{Applications of the Proposed Stability Metrics}

It is noted that none of these methods is by itself a necessary and sufficient condition for stability. Therefore, synergistic interpretation of these stability metrics need to be studied and applications of these stability metrics in controller A\&R, fault P\&D and flight software V\&V need to be demonstrated. 


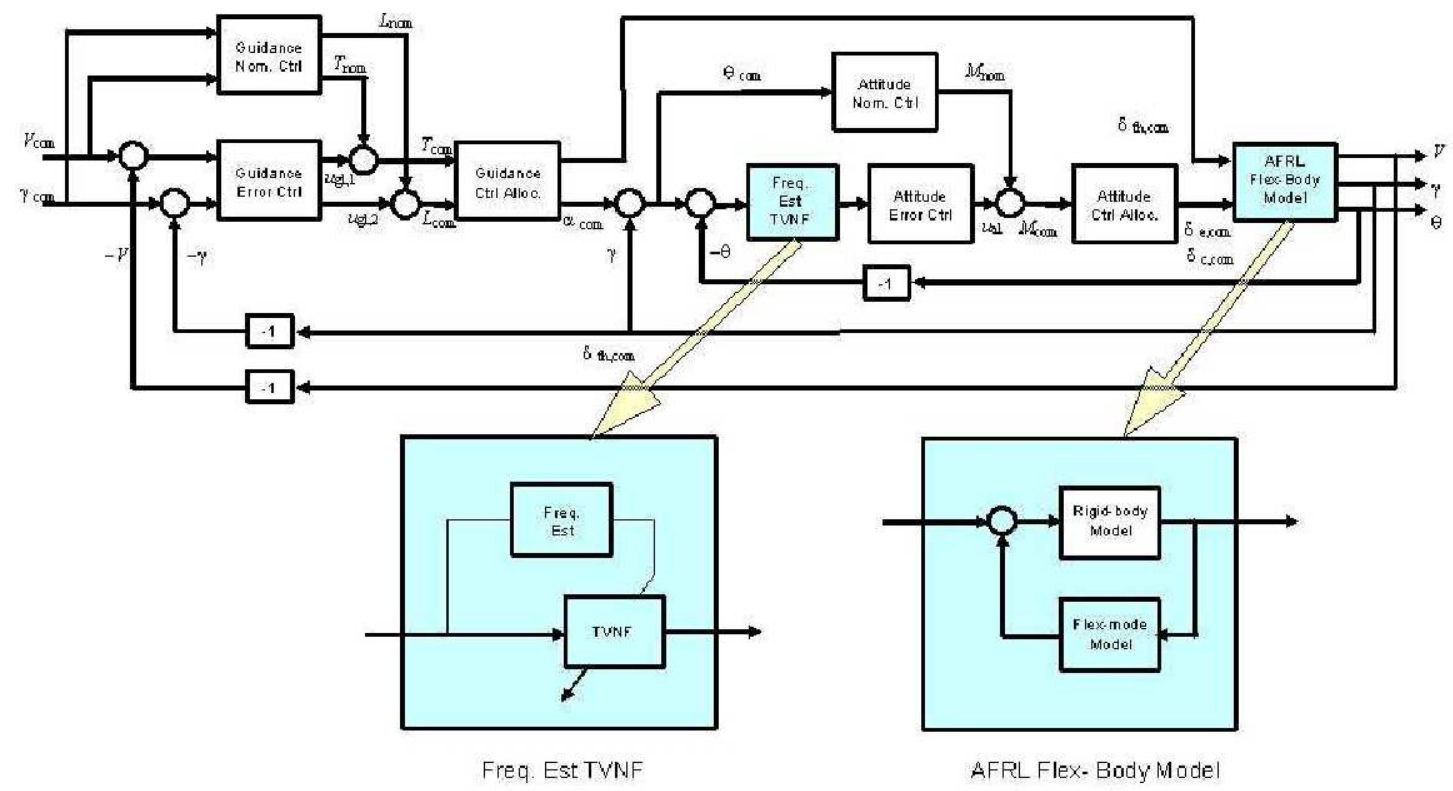

Figure 10. A 6-DOF Flight Controller Using TVNF Adaptation for Flex Mode Suppression

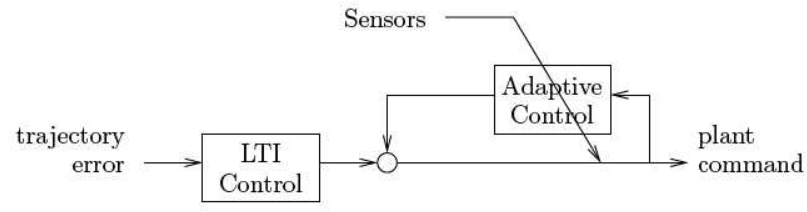

Figure 11. Multiplicative adaptive control assist architecture.

There are two basic approaches to control A\&R, direct and indirect. The former adjust the controller without explicit plant model parameter estimation or fault detection. One of such techniques is the timevarying bandwidth filters (TVBF) or time-varying notch filters (TVNF) for adaptive and fault tolerant control developed by Zhu et. al. ${ }^{21,22}$ The multi-band DI, PaM and $\mathcal{L}_{2}$-GM can be used to trigger control adaptation without explicitly identifying the plant parameters or faults to facilitate effective damping of excess kinetic energy and bleeding of excess potential energy. Figure 10 shows a 6-DOF flight controller utilizing the TVNF adaptation for a flexible hypersonic vehicle model developed by AFRL Air Vehicle Directorate $^{22}$ based on multi-band tracking error energy measurement, which can be modified to use the multi-band DT or PaM with potentially improved performance and robustness. An alternative approach is to use adaptive control involving neural networks as a parameterization of the control law where the adaptive portion of the controller may be used in a multiplicative (Figure 11) or additive (Figure 12) architecture. Adaptation design is performed based on Lyapunov stability criterion ${ }^{15,23,24}$ which may be modified to take advantage of the on-line estimated stability margins. For indirect adaptive and fault tolerant control schemes, parameter estimation or fault diagnosis will need to be explicitly performed.

The proposed stability metrics can used for fault P\&D in many ways. For example, the multi-band DI may provide modal information to indicate possible causes for the loss of stability - high frequency may indicate actuator failure, excitation of structural or aeroelastic modes, whereas low frequency may indicate failure in control effectors that hampers damping of vehicle kinetic energy. The multi-band PaMs indicate energy build up in different frequency band, which also provide hints to the cause and type of vehicle fault or flight condition. The correlation between the stability metrics and vehicle failures can be used by indirect adaptive or fault tolerant control schemes.

Finally, the proposed stability metrics may be useful in flight software V\&V in several ways. For example, 


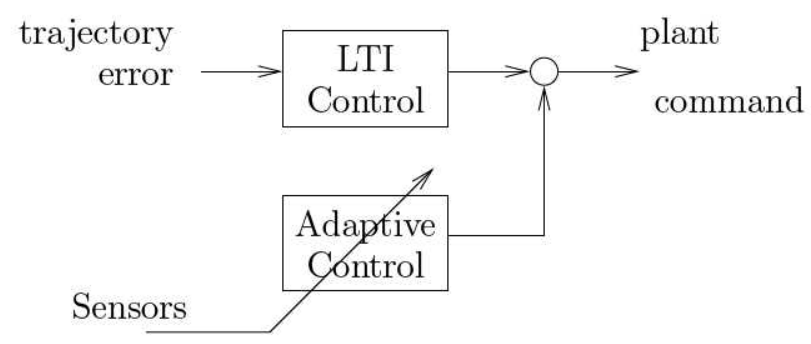

Figure 12. Additive adaptive control assist architecture.

once proving effective, adaptive flight software may be required to implement one or more stability metrics, and demonstrate that the software respond to these metrics in a predictable manner. The metrics can also be implemented in high fidelity, Monte Carlo simulations to validate adaptive flight software by exhaustive simulation test. The stability metrics can be used to specify requirement, or to demonstrate improvement in robustness and reliability.

\section{Real-time stability assessment by multi-resolution analysis}

Consider the $n$-dimensional NLTV tracking error or operating error dynamics

$$
\dot{x}=f(t, x), \quad x\left(t_{0}\right)=x_{0}, \quad t \geq t_{0}
$$

where $f$ is continuous in $t$ and Lipschitz in $x$, and $x(t) \equiv 0$ is the desired equilibrium state that needs to be stabilized. The time-dependence of $f$ may be due to either time-varying parameters or time-varying nominal tracking trajectory of the NLTV system. For simplicity, here we assume that (8) can be linearized at the operating (null equilibrium) point to a LTV system of the form

$$
\dot{x}=A_{c}(t) x, \quad x\left(t_{0}\right)=x_{0}, \quad t \geq t_{0}
$$

where $A_{t c}(t)$ is continuous and bounded, and is in the companion canonical form

$$
A_{c}(t)=\left[\begin{array}{cccc}
0 & & & \\
\vdots & & I_{n-1} & \\
0 & & & \\
-\alpha_{1}(t) & -\alpha_{2}(t) & \cdots & -\alpha_{n}(t)
\end{array}\right]
$$

where $I_{n-1}$ is the $(n-1) \times(n-1)$ identity matrix. By letting $y=x_{1}$, the state equation (9) is equivalent to an $n$ th-order scalar LTV differential equation

$$
\begin{gathered}
y^{(n)}+\alpha_{n}(t) y^{(n-1)}+\cdots+\alpha_{2}(t) \dot{y}+\alpha_{1}(t) y=0 \\
y^{(k-1)}\left(t_{0}\right)=x_{0_{k}}, k=1, \ldots n \quad t \geq t_{0}
\end{gathered}
$$

The general solution to LTV equation (10) can be written as

$$
y(t)=\sum_{k=1}^{n} C_{k} y_{k}(t)=\sum_{k=1}^{n} C_{k} e^{\int_{t_{0}}^{t} \rho_{k}(\tau) d \tau}
$$

where $\left\{y_{k}(t)=\exp \left(\int \rho_{k}(t) \mathrm{d} t\right)\right\}_{k=1}^{n}$ is a set of linearly independent solutions to (10), and $\left\{\rho_{k}(t)\right\}_{k=1}^{n}$ is called a parallel differential (PD) spectrum for (10) in which each $\rho_{k}(t)$ is called a $P D$-eigenvalue $e^{25,26}$ for $A_{\mathrm{c}}(t)$. The PD-eigenvalues are solutions to an $(n-1)$ th-order NLTV differential equation, called PD-characteristic equation. In particular, for $n=2$, the PD-character equation is given by the Ricatti equation ${ }^{\mathrm{b}}$

$$
\dot{\rho}+\rho^{2}+\alpha_{2}(t) \rho+\alpha_{1}(t)=0
$$

\footnotetext{
${ }^{\mathrm{b}}$ The general $n$ th-order PD-characteristic equation is omitted here for brevity, for details see Ref. ${ }^{25,26}$.
} 
Note that for real-valued coefficients $\alpha_{k}(t)$, the PD-eigenvalues $\rho_{k}(t)$ may be complex-valued in order to avoid singular solutions. Moreover, the synthesis formula for $\alpha_{k}(t)$ from a PD-spectrum $\left\{\rho_{k}(t)\right\}_{k=1}^{n}$ is given by

$$
\begin{aligned}
& \alpha_{1}(t)=\rho_{1}(t) \rho_{2}(t)+\frac{\rho_{1}(t) \rho_{\dot{2}}(t)-\rho_{2}(t) \dot{\rho}_{1}(t)}{\rho_{2}(t)-\rho_{1}(t)} \\
& \alpha_{2}(t)=-\rho_{1}(t)-\rho_{2}(t)-\frac{\dot{\rho}_{2}(t)-\dot{\rho}_{1}(t)}{\rho_{2}(t)-\rho_{1}(t)}
\end{aligned}
$$

The PD-eigenvalue concept is a natural extension of the eigenvalue concept for LTI systems in that, when the coefficients $\alpha_{k}(t) \equiv \alpha_{k}=$ constant, the PD-character (differential) equation (12) possesses constant solutions $\rho_{k}(t) \equiv \rho_{k}=$ constant that are conventional characteristic roots for $(10)$, and eigenvalues for $A_{\mathrm{c}}(t) \equiv A_{\mathrm{c}}$, for which (12) reduces to the conventional algebraic characteristic equation, and the synthesis formula (13) reduces to the conventional polynomial coefficient synthesis formula. Moreover, stability of the LTV system (9) or (10), and consequently the null equilibrium of the NLTV system (8), can be determined by the negativity of the real-part of the PD-eigenvalues. In particular, exponential stability is implied by $\operatorname{Re}\left(\rho_{k}(t)\right)<-c<0$, for some constant $c>0$, and for all $k$.

It is also noted that for each $k, \rho_{k}(t)$ can be written as

$$
\rho_{k}(t)=\frac{\dot{y}_{k}(t)}{y_{k}(t)}
$$

where $y_{k}(t)$ is complex-valued if $\rho_{k}(t)$ is. Thus $\rho_{k}(t)$ has a frequency dimension $t^{-1}$, which may be termed instantaneous frequency of $y(t)$. The notion of instantaneous frequency was original introduced for frequency modulated (FM) signals. It has been generalized to a much richer concept and extensively studied in the area of signal processing for signals generated by time-dependent processes, such as audio and visual signals ${ }^{27-29}$ However, the powerful tool of time-dependent frequency analysis, which is very pertinent to LTV and NLTV systems, has not been widely utilized in the area of control systems.

Figures 13 and 14 are taken from Ref. ${ }^{28}$ to illustrate the method of time-dependent frequency analysis for single-mode and multi-mode signals, respectively. The plots, called spectrograms, describe the harmonic distribution in the signal as function of time. These signals can be produced, respectively, by second-order and higher-order LTV systems, and there is an intrinsic relationship between the spectrogram and the PDeigenvalues of the LTV system that can be utilized for real-time stability assessment, as well as LTV system identification.

Consider a complex-valued single-mode signal

$$
x(t)=C \mathrm{e}^{j \int \omega(t) d t}
$$

This signal can be produced by a 2nd-order LTV system

$$
\ddot{x}-\frac{\dot{\omega}(t)}{\omega(t)} \dot{x}+\omega^{2}(t) x=0
$$

for which $\rho_{1,2}(t)= \pm j \omega(t)$ are PD-eigenvalues that constitutes a PD-spectrum. We now demonstrate the relationship between the spectrogram and the PD-eigenvalue by way of an example. Let $\omega(t)=-6 \sin (0.3 t)$, and denote by $\mathrm{P}_{\Delta}(t)$ the unit intensity pulse function centered at $t=0$ with magnitude $\Delta^{-1}$ and width $\Delta$, i.e.

$$
\mathrm{P}_{\Delta}(t)=\left\{\begin{array}{cl}
\frac{1}{\Delta} & t \in\left(-\frac{\Delta}{2}, \frac{\Delta}{2}\right] \\
0 & \text { elsewhere }
\end{array}\right.
$$

Let $\widehat{x}(t)$ be a function composed of complex sinusoidal splines

$$
\widehat{x}(t)=\sum_{k=-\infty}^{\infty} P_{\Delta}(t-k \Delta) \mathrm{e}^{j \omega_{k} t} \Delta
$$




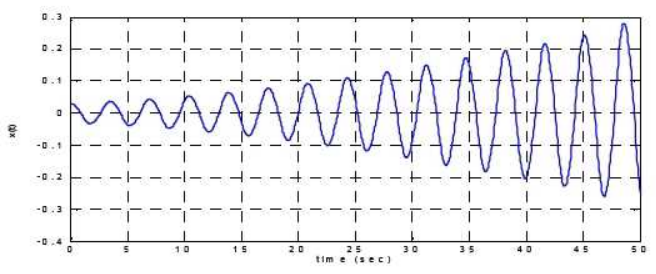

(a) Time trajectory of $\theta(t)$

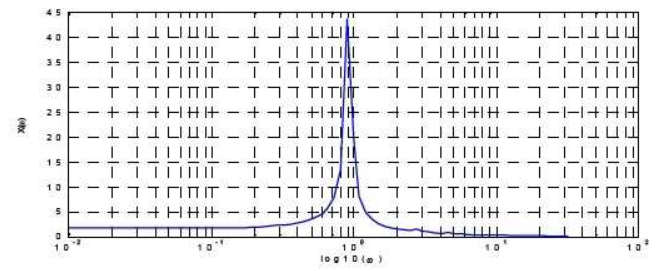

(b) Spectrum of $\theta(t)$ over $[0,100]$

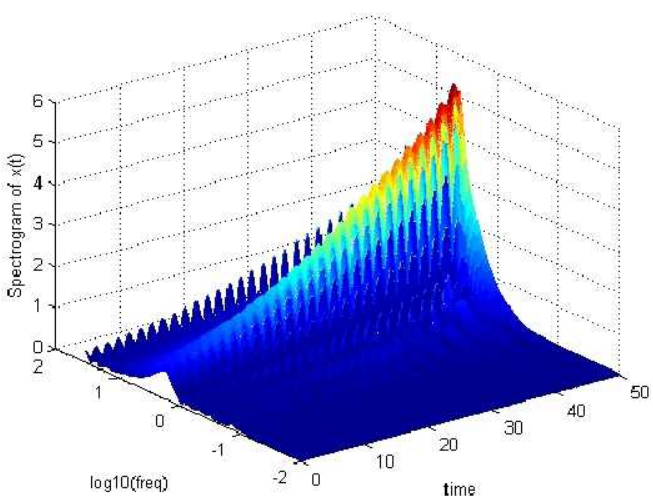

(c) Spectrogram of $\theta(t)$

Part (a) shows the time trajectory $\theta(t)$ of a playground swing discussed in this paper. The oscillation not only diverges in magnitude, but also decrease in frequency. The Fourier spectrum shown in Part (b) does not capture either of these important features of the oscillatory mode. But these features are captured by the Spectrogram shown in Part (c), see also Figures 3 and 17 and the discussions thereof.

Figure 13. Spectrogram of a single-modal signal.

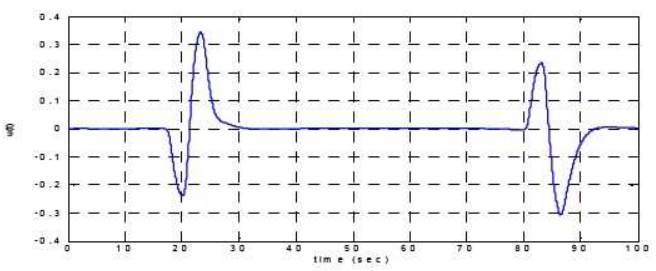

(a) Time trajectory of $u(t)$

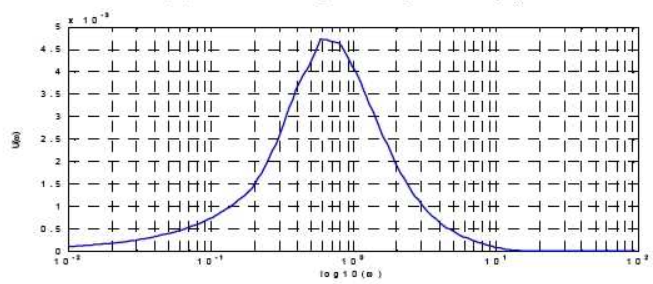

(b) Spectrum of $u(t)$ over $[0,100]$

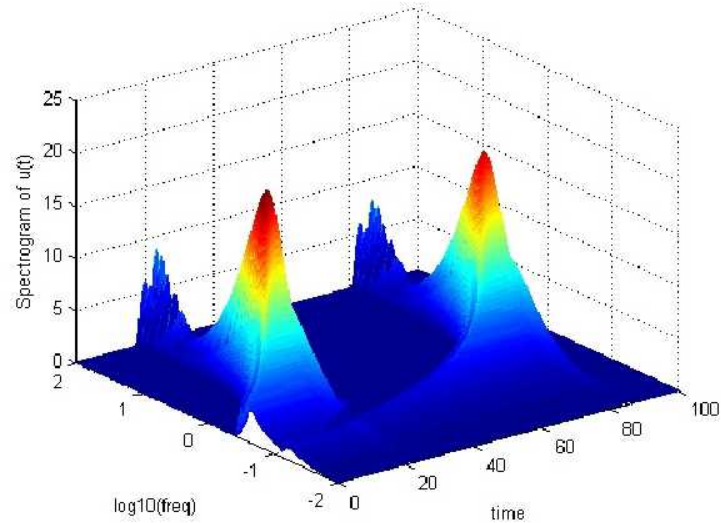

(c) Spectrogram of $u(t)$

Part (a) shows the tracking error of the body frame velocity $u(t)$ along the $x$-axis of a 6DOF vertical take-off and landing (VTOL) UAV when it flies a semicircular trajectory. The doublet tracking error is due to the acceleration and deceleration at the end points of the semicircular trajectory. The simulation consists of 66th-order dynamics. Part (b) shows the Fourier transform of the tracking error signal over the 100 -second time interval. The spectrogram in Part (c) not only shows the energy concentration in the frequency domain with much higher resolution, but also the time when the energy built-up happened, and the exponential convergence of the tracking error.

Figure 14. Spectrogram of a multi-modal signal. 
where $\omega_{k}=\omega(k \Delta)$. For sufficiently small $\Delta$, the piecewise constant function

$$
\widehat{\omega}(t)=\omega_{k}, \quad t \in\left(\frac{2 k-1}{2} \Delta, \frac{2 k+1}{2} \Delta\right]
$$

is a piecewise constant approximation of $\omega(t)$, and $\widehat{x}(t)$ is a sinusoidal spline approximation of $x(t)$. Now taking the short time Fourier transform (STFT) of $\widehat{x}(t)$ with the $k$ th analysis (observation) window $\mathrm{P}_{\Delta}(t-k \Delta)$, we have

$$
\hat{X}_{k}(j \omega)=\operatorname{sinc}\left(\frac{\Delta}{2}\left(\omega-\omega_{k}\right)\right)
$$

Figure 15 shows the plots of $\omega(t), \widehat{\omega}(t)$ and $\left|\hat{X}_{k}(j \omega)\right|$ for different analysis window sizes. It is noted that the collection of $\left|\hat{X}_{k}(j \omega)\right|$ constitute a spectrogram for $x(t)$, where

$$
\max _{\omega}\left|\hat{X}_{k}(j \omega)\right|=\Delta=\left|\hat{X}_{k}\left(j \omega_{k}\right)\right|
$$

with main lobe bandwidth

$$
B_{m}(\Delta)=4 \pi / \Delta
$$

Thus, we can estimate the (imaginary part of) PD-eigenvalue from the modal peaks of the spectrogram at $t=k \Delta$. In order to improve the accuracy of the estimate $\widehat{\omega}(t)$, it is desirable to use smaller time window size $\Delta$. However, as $\Delta$ decreases, the spectral peak is flattened, making an accurate reading the center frequency more difficult. In fact, as $\Delta \rightarrow 0$,

$$
\begin{gathered}
\max _{\omega}\left|\hat{X}_{k}(j \omega)\right| \rightarrow 0 \\
B_{m}(\Delta)=4 \pi / \Delta \rightarrow \infty
\end{gathered}
$$

i.e. the STFT spectrum $\left|X_{k}(j \omega)\right|$ approaches the constant zero "centered" at $\omega_{k}$, as can be seen in Figures $15(\mathrm{~b})$ and 15(c). However, as $\Delta \rightarrow 0, \mathrm{P}_{\Delta}(t) \rightarrow \delta(t)$, it follows

$$
\begin{gathered}
\widehat{\omega}(t) \rightarrow \omega(t) \\
\widehat{x}(t) \rightarrow \int_{-\infty}^{+\infty} \delta(t-\tau) \mathrm{e}^{j \int \omega(\tau) d \tau} \mathrm{d} \tau=x(t)
\end{gathered}
$$

This shows that $\omega(t)$ is in fact the precise measurement of the instantaneous frequency at the instant $t$ (see Figure 15c). Thus it is highly desirable to develop methods that directly estimate the instantaneous frequency from the signal $x(t)$, and then construct an accurate spectrogram from the PD-spectrum.

The signal $x(t)$ in this example does not decay with time. For signals whose amplitude envelope decays (or grows) with time, the PD-eigenvalue will have a real part, which can be estimated by looking at the peak amplitude of the spectrogram, as demonstrated in the sequel by the swing and jigsaw pendulum examples using the MRA method.

The imaginary part $\omega_{d}(t)$ of the PD-eigenvalues $\rho(t)=\sigma(t) \pm j \omega_{d}(t)$ can be identified by connecting the peak magnitudes of the frequency spectrum at each time $t$, and the real part $\sigma(t)$ can be identified by calculating the slope of $\ln \left(X_{\psi}(\omega, t)\right)$ at each time $t$. 


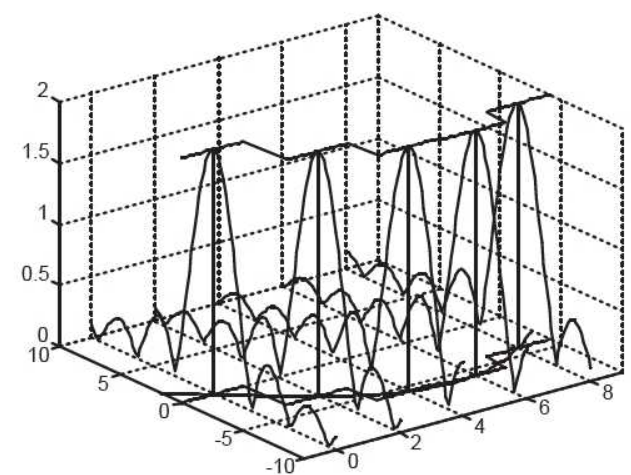

(a) Large Observation Window, $\Delta=2.0$, $\widehat{\omega}(t)=-6 \sin (0.6 k), \quad 2 k-1<t \leq 2 k+1$, $\left|\hat{X}_{k}(j \hat{\omega})\right|=2 \operatorname{sinc}\left(\omega-\omega_{k}\right), \quad B_{m}(\Delta)=2 \pi$

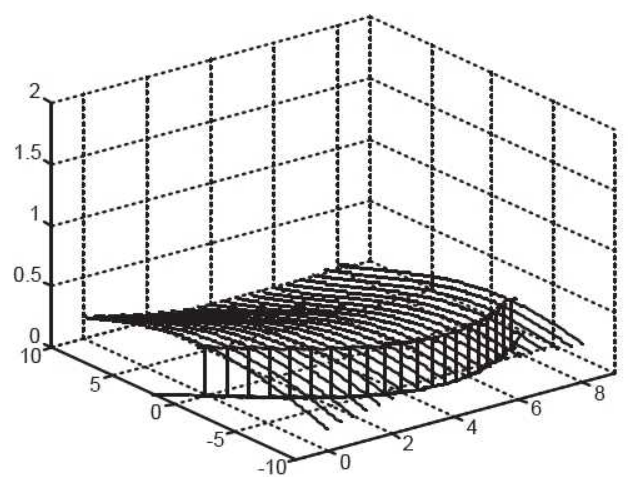

(b) Small Observation Window $\Delta=0.4$ $\widehat{\omega}(t)=-6 \sin (0.12 k), \quad 0.4 k-0.2<t \leq 0.4 k+0.2$ $\left|\hat{X}_{k}(j \hat{\omega})\right|=0.4 \operatorname{sinc}\left(0.2\left(\omega-\omega_{k}\right)\right), \quad B_{m}(\Delta)=10 \pi$

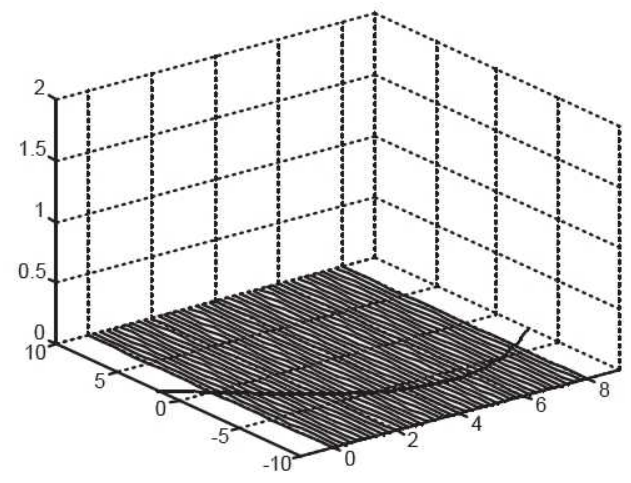

(c) Observation Window $\Delta \rightarrow 0, k \Delta \rightarrow t$ $\widehat{\omega}(t)=-6 \sin (0.3 k \Delta) \rightarrow-6 \sin (0.3 t)=\omega(t)$ $\left|\hat{X}_{k}(j \hat{\omega})\right|=\Delta \operatorname{sinc}\left(\frac{\Delta}{2}\left(\omega-\omega_{k}\right)\right) \rightarrow 0, \quad B_{m}(\Delta)=\frac{4 \pi}{\Delta} \rightarrow \infty$

Figure 15. Relationship between the STFT spectrogram and PD-eigenvalues, $\omega(t)=-6 \sin (0.3 t)$. 
Mother wavelet for stability analysis $(a=5)$

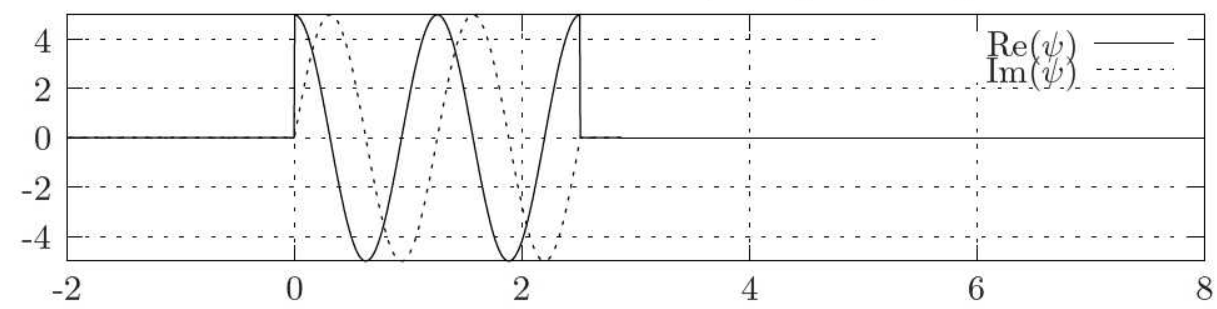

Fourier transform of mother wavelet, $a=5$

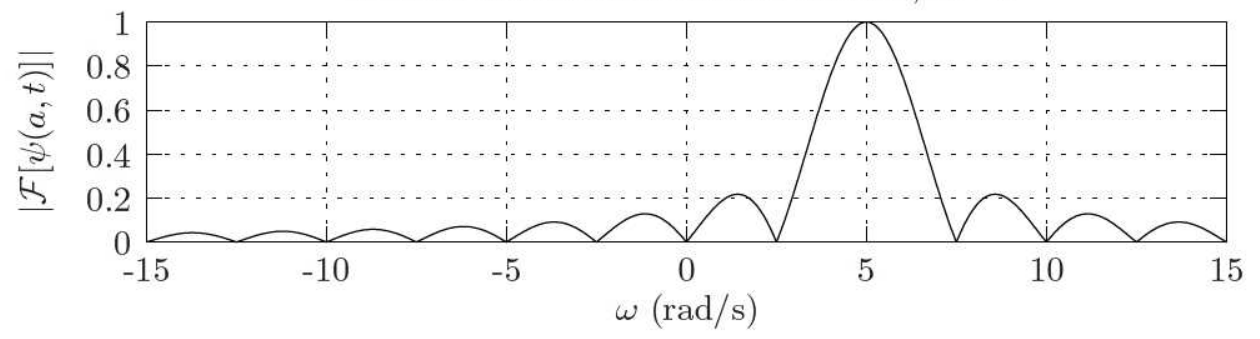

Figure 16. Mother wavelet used for stability analysis.

\section{Case study}

In this section we present a preliminary case study of real-time stability assessment for NLTV systems by applying one of the proposed approaches, namely, Lyapunov first method, to the playground swing and jigsaw mounted inverted pendulum examples to demonstrate the effectiveness of the proposed approaches over the conventional stability metrics. The study is facilitated by multi-resolution (wavelet) analysis (MRA) of the tracking or operating error state trajectory, which is a useful technique that is applicable to both trajectory based (Lyapunov first) and energy based (Lyapunov second and passivity) stability assessment. We demonstrate the utility of this method with the systems examined in Example 1 (pumped swing) and Example 2 (jigsaw).

For this case study, define the family of wavelets

$$
\psi(a, t)= \begin{cases}a e^{j a t} & t \in\left[0, \frac{4 \pi}{a}\right] \\ 0 & \text { otherwise }\end{cases}
$$

with corresponding Fourier transform

$$
\Psi(a, \omega)=\mathcal{F}[\psi(a, t)]=e^{j 2 \pi / a} \operatorname{sinc}\left(\frac{2 \pi(a-\omega)}{a}\right)
$$

(see Figure 16). Given a signal $x(t)$ we define its wavelet transform

$$
X_{\psi}\left(\omega, t_{0}\right)=\left\langle\psi\left(\omega, t-t_{0}\right), x(t)\right\rangle=\int_{-\infty}^{\infty} x(t) \psi\left(\omega, t-t_{0}\right) d t
$$

(Recall that an inner product in the time domain corresponds to a multiplication operation in the frequency domain, with appropriate scaling for phase information.)

Recall for these systems in Examples 1 and 2 that frozen time stability analysis of the swing model predicts that it is stable. However, as seen in Figure 17, this prediction is incorrect. The system response, shown in the upper left plot, shows an increasingly large oscillation throughout the first 50 seconds of operation. The upper right plot is a multiresolution analysis of the system response, a plot of $\log _{10}\left|X_{\psi}\right|$ as a function of time and frequency. The "height" of the peak of the MRA is shown in the lower left plot, and provides a local measure of exponential/damping behavior of the system - that is, an assessment of stability. The frequency associated with this peak is marked in white in the lower right plot. That is, wavelet analysis permits the 

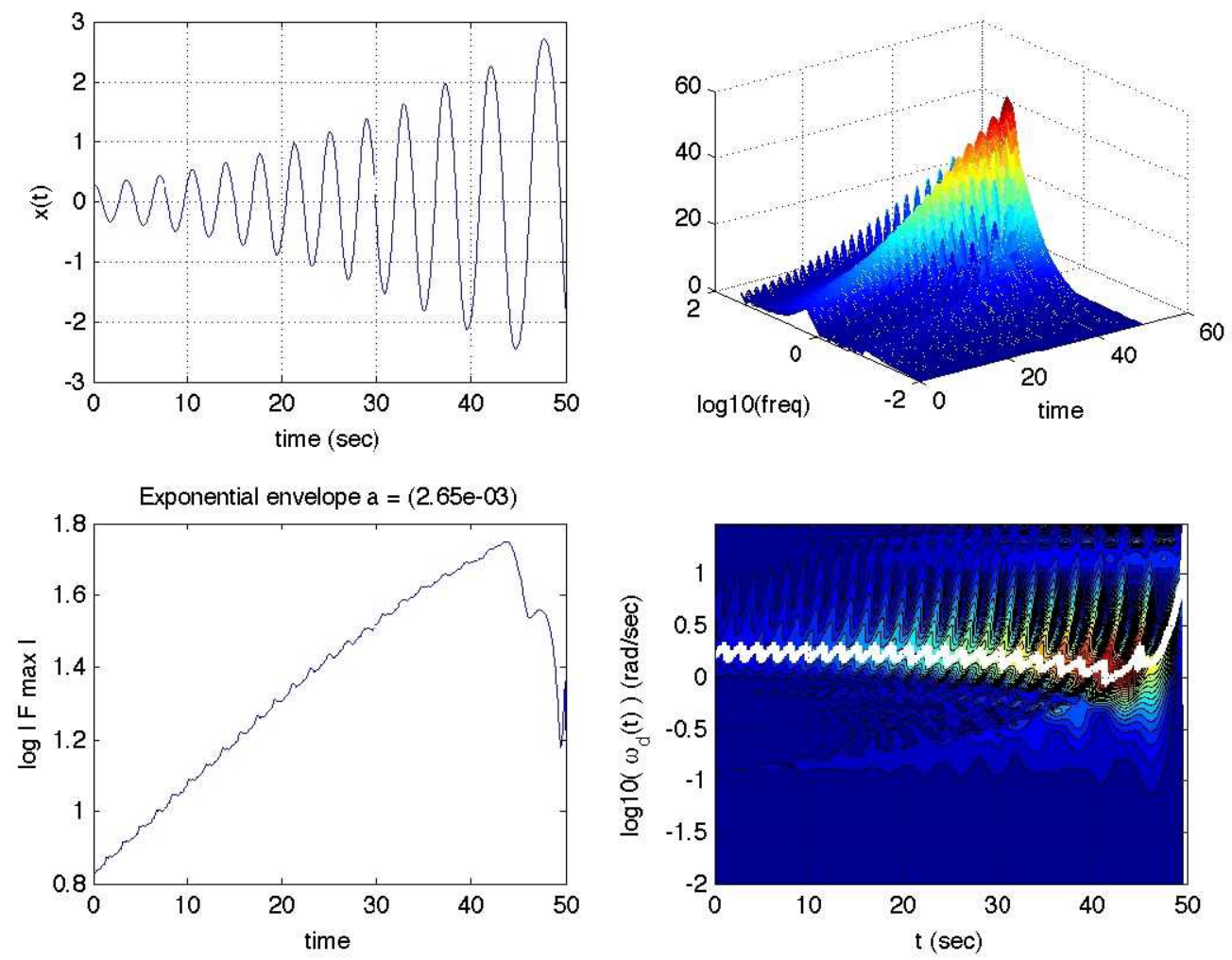

Figure 17. Multiresolution analysis analysis of swing behavior. LTI analysis predicts stability; wavelet analysis quickly detects instability.

identification of the time-varying frequency and exponential envelope that describe the instability. Subject to the limits of Heisenberg's uncertainty principle ${ }^{30}$ this MRA identifies time-varying frequency and damping characteristics of the system response.

Conversely, frozen time stability analysis of the jigsaw model Example 2 predicts that it will be unstable. However, as seen in the upper left plot in Figure 18, the system response in this simulation appears to behave as a damped sinusoid. Multi-resolution analysis (see the upper right plot of Figure 18 reveals the frequency $\omega_{0}$ of oscillation and, in turn, the slope of $\log \left|X_{\psi}\left(\omega_{0}, t\right)\right|$ reveals that the system can be well approximated by a pair of complex conjugate poles with negative real parts. Multi-resolution analysis also detects a nigh frequency oscillation in the first few seconds of simulation, which is also visible on the time-domain plot (upper left).

Remark 1. The MRA spectrogram method for real-time stability assessment presented herein can be extended to higher order (multi-mode) systems by first isolate the modes using bandpass filters, followed with single-mode analysis. This method is applicable to LTV and linearizable NLTV systems. However, for NLTV systems, the nonlinearity may cause spectral distortion by creating higher harmonics or sub-harmonics that may be mistaken as additional modes. This may cause problem for modal analysis, but it should not be a problem for energy based method, as passive nonlinearity does not inject energy into the signal.

Remark 2. It is noted that while the spectrogram analysis can be used to assess stability in real-time using the PD-eigenvalues estimated from the real-time measurement of a particular state trajectory $x(t)$, it is not reliable to ascertain stability of the null equilibrium based just on this one measurement. There may be dynamic modes that are not excited in a particular trajectory. Thus, to assess stability of the equilibrium, all modes need to be persistently excited. Moreover, for time-varying systems, it is important to determine uniform stability. However, this would require to test the system for infinitely large initial time $t_{0}$, which is not practical. But this should not be a problem for engineering applications as all real world systems have a finite mission duration, and we may assume the system parameters freeze at the mission terminal time.

Remark 3. With persistent excitation, the spectrogram analysis can be used to identify a model for a LTV 

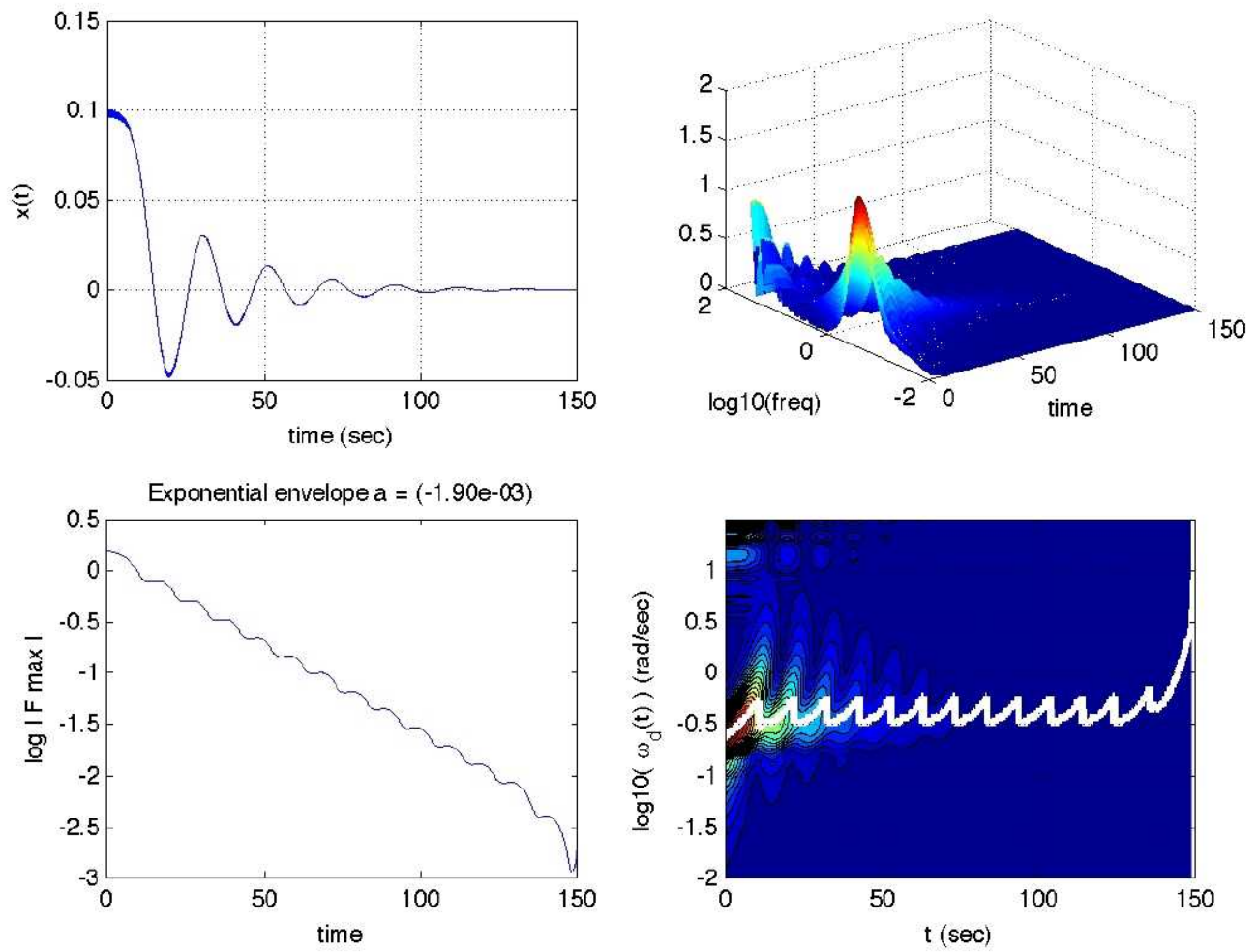

Figure 18. Wavelet analysis of jigsaw behavior. LTI analysis predicts instability; wavelet analysis detects stability.

system using the estimated PD-spectrum. It is imperative to use the correct synthesis formulation to obtain the time-varying parameters for the identified LTV system. In fact, any frequency domain LTI spectral identification algorithm can be used to estimate the PD-eigenvalues at frozen time moment from the spectrogram. The fundamental difference between the (correct) PD-spectral synthesis and the (unreliable) frozen-time method is that the latter uses the algebraic polynomial synthesis formula on time-varying eigenvalues, which introduces errors that are dependent on the rate of change of the PD-eigenvalues. This may explain why the froze-time based gain-scheduling, robust and adaptive control techniques have been successful for slowly varying systems, but are challenged by fast varying systems.

Remark 4. In the example illustrating the relationship between the spectrogram and PD-eigenvalues, it is noted that a large time window (step) yields poor time resolution, but better frequency resolution, while a smaller time window provides a better time resolution but poor frequency resolution. This phenomenon is known as the uncertainty principle for the STFT. While the MRA method improves the STFT by using smaller time-windows for higher frequency harmonics, the ultimate solution, as shown in Figure 15, is to directly compute the PD-eigenvalues using equation (14). However, the difficulty in this method is that, for complex modes, the imaginary part of the real-valued signal must be estimated. This may be accomplished using the Hilbert transform. However, Hilbert transform is an anti-causal operation that cannot be applied to real-time signals. Therefore, approximations must be made, a subject that deserves future study. Alternatively, a quadrature signal, i.e. another solution trajectory that is linearly independent of the measured trajectory can be constructed to facilitate the estimate of PE-eigenvalues using (14).

Remark 5. The MRA spectrogram real-time stability assessment method presented herein is based on Lyapunov's first method (spectral method). However, the spectrogram can also be used to facilitate stability assessment using energy-based stability criteria, such as Lyapunov's second method and passivity methods. This is based on the Parseval principle that the energy contained in a signal is the same in its time-domain and frequency domain representations. 


\section{Conclusions}

The need in aerospace systems for reliable operation in high-performance operating conditions has led to increased interest in the use of NLTV (e.g., adaptive or reconfigurable) control laws for these systems. Adaptive control laws, while based in Lyapunov stability theory, do not easily fall into existing theory for verification and validation $(\mathrm{V} \& \mathrm{~V})$, and thus can be considered a risk in an overall design. The weaknesses of classical LTI stability assessment are shown in two simulation examples, one of a playground swing and the second of a jigsaw.

In this paper, we provide a roadmap to the development of stability metrics appropriate for use in V\&V and in on-board stability assessment. The conceptual basis for these metrics is drawn from well established stability concepts such as the small gain theorem, Lyapunov's first and second methods, and passivity theory. We also present a multi-resolution analysis approach that provides real-time assessment of stability in each of the example systems. The results of this analysis are closely tied to LTV system concepts (PD-eigenvalues, etc.) and are easily interpreted.

\section{References}

${ }^{1}$ Doyle, J., Glover, K., Khargonekar, P., and Francis, B., "State-Space Solutions to Standard $H_{2}$ and $H_{\infty}$ Control Problems," IEEE Transactions on Automatic Control, Vol. AC-34, No. 8, 1989, pp. 831-847.

${ }^{2}$ Balas, G., Doyle, J., Glover, K., Packard, A., and Smith, R., $\mu$-Analysis and Synthesis Toolbox: A User's Guide, The MathWorks, 1991.

${ }^{3}$ Vidyasagar, M., "The graph metric for unstable plants and robustness estimates for feedback stability," IEEE Transactions on Automatic Control, Vol. AC-29, 1984, pp. 403-418.

${ }^{4}$ El-Sakkary, A. K., "The Gap Metric: Robustness of Stabilization of Feedback Systems," IEEE Transactions on Automatic Control, Vol. AC-30, No. 3, March 1985, pp. 240-247.

${ }^{5}$ Qiu, L. and Davison, E. J., "Pointwise Gap Metrics on Transfer Matrices," Proceedings of the $29^{\text {th }}$ Conference on Decision and Control, No. TP-13, Honolulu, Ha, 1990, pp. 2431-2436.

${ }^{6}$ Anderson, B. D. O. and Brinsmead, T., "Additive, Multiplicative, and Inverse Multiplicative Robust Stability: Connections with the $\nu$-Gap Metric," Proceedings of the $40^{\text {th }}$ IEEE Conference on Decision and Control, No. 3069-3074, Orlando, FL, 2001.

${ }^{7}$ Hsieh, G. C. and Safonov, M. G., "Conservatism of the Gap Metric," IEEE Transactions on Automatic Control, Vol. 38, No. 4, 1993, pp. 594-598.

${ }^{8}$ Cusumano, S. J. and Poolla, K., "Nonlinear Feedback Versus Linear Feedback For Robust Stabilization," Proc. 27th Conference on Decision and Control, IEEE, Austin, Tx., 1988, pp. 1776-1780.

${ }^{9}$ maryam1960, "Global Stability Criteria for Differential Systems," J. Osaka Math., Vol. 12, 1960, pp. 305-317.

${ }^{10} \mathrm{Wu}$, M. Y., "A Note on Stability of Linear time-varying systems," IEEE Trans. Automatic Control, Vol. AC-19, 1974, pp. 162.

${ }^{11}$ Zhu, J., "A note on extension of the eigenvalue concept," IEEE Control Systems Magazine, Vol. 13, Dec. 1993, pp. 68-70.

${ }^{12}$ Richards, J. A., Analysis of Periodically Time-Varying Systems, Springer-Verlag, 1983.

${ }^{13}$ Khalil, H. K., Nonlinear Systems, Prentice-Hall, Upper Saddle River, NJ, 3rd ed., 2002.

${ }^{14}$ Cai, X.-S. and Han, Z.-Z., "Robust Stabilization and Passivity of Nonlinear Systems With Structural Uncertainty," IEE Proc.-Control Theory Appl., Vol. 153, No. 6, Nov. 2006, pp. 641-646.

${ }^{15}$ Lewis, F. L., Yeşildirec, A., and Liu, K., "Multilayer Neural Net Robot Controller with Guaranteed Tracking Performance," IEEE Transactions on Neural Networks, Vol. 7, No. 2, Mar. 1996, pp. 388-399.

${ }^{16}$ Best, E., Adami, T., and Zhu, J., "Instability Detection for Nonlinear Time-Varying Systems Using Lyapunov Exponent," Proceedings AIAA Guidance, Navigation and Control Conference, No. AIAA-2003-5359, Austin, TX, 2003.

${ }^{17}$ Lyapunov, A. M., The General Problem of the Stability of Motion, CRC, Bristol, PA, 1992.

${ }^{18}$ Rozgonyi, S., Hangos, K., and Szederkenyi, G., "Improved Estimation Method of Region of Stability for Nonlinear Autonomous Systems," 2006.

${ }^{19} \mathrm{Zhu}$, J. and Mickle, M. C., "Synthesis of time-varying bandwidth filters based on all-pole LTI prototypes," Proceedings of the American Control Conference, Philadelphia, PA, 1998, pp. 2889-2894.

${ }^{20}$ Adami, T. M., Sabala, R., and Zhu, J. J., "Time-varying notch filters for control of flexible structures and vehicles," Proceedings, $22^{\text {nd }}$ Digital Avionics Systems Conference, Indianapolis, Indiana, Oct. 2003.

${ }^{21}$ Zhu, J., Lawrence, D., Fisher, J., Shtessel, Y., Hodel, A., and Lu, P., "Direct Fault Tolerant RLV Attitude Control A Singular Perturbation Approach," AIAA Conference on Guidance, Navigation, and Control, Monterrey, CA, 2002, Paper 2002-4778.

${ }^{22}$ Adami, T. and Zhu, J. J., "Control of a Flexible, Hypersonic Scramjet Vehicle using a Differential Algebraic Approach," Proceedings, AIAA Guidance, Navigation and Control Conference, No. AIAA-2007-6328, Hilton Head, SC, Aug. 2007.

${ }^{23}$ Johnson, E. N. and Calise, A. J., "Neural Network Adaptive Control of Systems with Input Saturation," Proceedings of the American Control Conference, Arlington, VA, Jun 25-27 2001, pp. 3527-3532.

${ }^{24}$ McFarland, M. B. and Calise, A. J., "Adaptive Nonlinear Control of Agile Antiair Missiles Using Neural Networks," IEEE Transactions on Control Systems Technology, Vol. 8, No. 5, Sep 2000, pp. 749-756. 
${ }^{25}$ Zhu, J. and Johnson, C. D., "Unified Canonical Forms for Matrices Over a Differential Ring," Linear Algebra and Its Applications, 1991, pp. 201-248.

${ }^{26} \mathrm{Zhu}$, J., "PD-spectral theory for multivariable linear time-varying systems," Proceedings $36^{\text {th }}$ IEEE Conference on Decision and Control, San Diego, CA, Dec. 1997, pp. 3908-3913.

${ }^{27}$ Boashash, B. and Jones, G., "Instantaneous Frequency and Time-Frequency Distributions," Time-Frequency Signal Analysis-Methods and Applications, edited by B. Boashas, Halsted Press, John Wiley \& Sons, 1992, pp. 43-73.

${ }^{28}$ Cohen, L. and Lee, C., "Instantaneous Bandwidth," Time-Frequency Signal Analysis-Methods and Applications, edited by B. Boashash, Halsted Press, John Wiley \& Sons, 1992, pp. 98-117.

${ }^{29}$ Cohen, L., Time-Frequency Analysis, Prentice Hall, Inc., 1995.

${ }^{30}$ Strang, G. and Nguyen, T., Wavelets and Filter Banks, Wellesley Cambridge Press, wellesley, mass. ed., 1996. 